\title{
An improved MULTIMOORA method for multi-valued neutrosophic multi-criteria group decision-making based on prospect theory
}

Fei Xiao ${ }^{1}$, Jing Wang ${ }^{2,3}$, Jian-qiang Wang ${ }^{4}$

1. School of Business, Central South University, Changsha 410083, PR China

Tel: (+86) 73188830594, Email: xiaofei2017@csu.edu.cn

2. College of Tourism, Hunan Normal University, Changsha 410081, PR China

3. College of Logistics and Transportation, Central South University of Forestry and Technology, Changsha 410004, PR China

Tel: (+86) 73185623292, Email: wangjing@csuft.edu.cn

4. School of Business, Central South University, Changsha 410083, PR China

Tel: (+86) 73188830594, Email: jqwang@csu.edu.cn

Correspondence should be addressed to Jing Wang: wangjing@ @suft.edu.cn

\begin{abstract}
At present, there are many subways being constructed in many cities. In the construction of subways, an appropriate scheme is helpful to save cost and ensure the quality of the project. This paper attaches great importance to present a multi-criteria group decision-making (MCGDM) method to deal with selecting an appropriate construction scheme for subways. The process of selecting an appropriate construction scheme for subways is complex because it includes a great deal of fuzzy and uncertain information which can be presented by multi-valued neutrosophic numbers (MVNNs). In addition, in order to handle the interaction of inputs, an improved generalized multi-valued neutrosophic weighted Heronian mean (IGMVNWHM) operator is introduced. Subsequently, a new distance measure between two MVNNs is defined for deriving the objective criteria weights. Considering that decision-makers are not completely rational, we develop an improved multi-valued neutrosophic MULTIMOORA method based on prospect theory. Finally, an application example of selecting an appropriate construction scheme for a subway and the influence of parameter are described. In addition, the validity and advantages of the proposed method are proved by comparative analysis.
\end{abstract}

Keywords: multi-criteria group decision-making, Heronian mean operator, MULTIMOORA, prospect theory, multi-valued neutrosophic sets

\section{Introduction}

Owing to quality of life improvements, a great deal of families have their own cars, and the problem of traffic jams grows more prominent. Subways have some advantages, such as convenience, speediness and 
punctuality. Furthermore, selecting an appropriate construction scheme for subways is related to multi-criteria group decision-making (MCGDM). There are several criteria that should be considered, such as technology level, environmental condition, public intervention risk, and force of supervision. Existing studies for selecting suitable construction schemes often utilize real numbers to represent criteria values [1-3] However, the uncertainty and intricacy of our real decision problems are a significant improvement, thus, the expression of evaluation information should be changed. We cannot present decision information only in crisp numbers any longer.

To grapple with uncertainty and vagueness, Zadeh presented fuzzy sets [4]. Since then, there have been a great number of achievements in fuzzy sets [5-9]. However, fuzzy sets only consider membership functions, they cannot tackle more complex problems. Therefore, Atanassov presented intuitionistic fuzzy sets (AIFSs) [10-12], which include membership and non-membership degrees. AIFSs have been employed in neural networks [13, 14] and medical diagnoses [15]. Thereafter, AIFSs were employed in interval-valued intuitionistic fuzzy sets (AIVIFSs) [16]. Nevertheless, AIFSs and AIVIFS only solve the fuzzy information whose membership and non-membership degrees have only one specific number. For example, whether the decision-makers think a statement is true may be 0.5 or 0.6 . To tackle this problem, hesitant fuzzy sets (HFS) were presented [17-19]. Afterward, the generalized hesitant fuzzy and dual hesitant fuzzy sets (DHFSs) were produced $[20,21]$.

Though AIFSs have been improved since their conception, they are not able to handle all kinds of uncertainties in real decision-making. Inconsistent and incomplete information cannot be approached by AIFSs. For example, decision-maker may hold that the statement is true (0.4) or that the statement is false (0.3), but the expert may also be unsure (0.3) [22]. To solve this problem, neutrosophic sets (NSs) were presented $[23,24]$. At first, each section of NSs lies in $] 0^{-}, 1^{+}[[25,26]$. However, it is difficult to utilize NSs in practice. Therefore, Sahin and Kucuk [27] presented single-valued neutrosophic sets (SVNSs). What's more, a great deal of achievements have been accomplished in single-valued neutrosophic sets [28-30].

However, decision-makers may be hesitant to provide every attribute value for each membership on SVNSs. For example, an expert may think statement is true ( 0.5 or 0.6$)$, it is false $(0.2$ or 0.3$)$ or they may be unsure $(0.3$ or 0.4). Multi-valued neutrosophic sets (MVNSs) can be used to handle this problem. Liu et al. [31] firstly defined MVNSs and, at the same time, the weighted average (WA) and weighted geometric (WG) operator had been expressed. Then, Peng et al. [32] proposed a multi-valued neutrosophic qualitative flexible (QUALIFLEX) approach for MCDM. For the construction scheme selection of subways, the expert group 
have different opinions due to their distinct professional backgrounds, and they may be hesitant to give each alternative evaluation information. Therefore, in this paper, we utilize MVNSs to depict the evaluation information.

The aggregation operators play an important role in MCDM problem and a lot of researches have been produced in this field. The most commonly used aggregation operators are weight average (WA) operator and weight geometry (WG) operator $[33,34]$. Furthermore, the interrelations of evaluation values should be taken into consideration. Several operators which can deal with this problem have been presented, such as power average (PA) operator [35] and Bonferroni mean (BM) operator [36]. Similar to these operators, Heronian mean (HM) operator has the same function. Numerous researchers explored HM operators [37, 38]. Liu et al. [39] brought HM operators into NSs to expand the scope of its use. Then, based on the lack of idempotency in existing HM operators, Peng et al. [40] introduced the improved generalized weighted HM (IGWHM) operator and improved generalized weighted geometric HM (IGWGHM) operator. Furthermore, HM operators were extended into neutrosophic hesitant fuzzy sets [41]. To deal with some unreasonable evaluation values, Liu et al. [42] combined power operators with HM operators. Considering the interactions between experts when selecting construction scheme for subways, it is necessary to introduce HM operator into this MCGDM problem.

In the process of practical application, the theoretical methods related to MCGDM often encounter the same problem, and the evaluation results obtained by different evaluation methods are different. This kind of problem about the robustness of decision-making analysis has attracted the attention of many scholars [43]. In order to gather the advantages of different evaluation methods, MULTIMOORA method was proposed [44]. Because the MULTIMOORA method includes three different decision-making methods, MULTIMOORA method is more robust than MOORA method [45]. At present, the MULTIMOORA method has been extended into AIFSs [46], HFS [47] and NSs [48]. It also has been applied into personnel selection [49], supplier selection [50] and quality management [51], etc. But, existing studies about MULTIMOORA method seldom consider the bounded rationality of decision-makers and has not been extended into MVNSs.

On the basis of the above analysis, the contributions of our research are listed below:

(1) MVNSs are adopted to express the assessments of decision-makers, and improved generalized multi-valued neutrosophic weighted Heronian mean (IGMVNWHM) operator is presented for aggregating the evaluation matrix to deal with the interactions of inputs.

(2) A new distance measure between two multi-valued neutrosophic numbers (MVNNs) is defined. Then, a distance-based method for deriving the objective criteria weights is developed. 
(3) This paper extends the MULTIMOORA method into MVNSs. In addition, we present an improved multi-valued neutrosophic MULTIMOORA method based on prospect theory (IMVN-PT-MULTIMOORA). This method can solve practical decision-making problem effectively because it considers that the decision-makers are not completely rational.

The rest of the paper is organized below. In Section 2, some basic theories are stated. In Section 3, IGMVNWHM operator, distance measure and IMVN-PT-MULTIMOORA method are presented. Subsequently, a solution framework for multi-valued neutrosophic MCGDM (MVN-MCGDM) problem is presented in Section 4. In Section 5, illustrative example, influence of the parameter analysis and comparative analysis are conducted. In Section 6, some conclusions are drawn.

\section{Preliminaries}

\subsection{MVNSs}

Definition $1[52,53]$. Let $X$ be a space of points (objects), with a generic element in $X$ denoted by $x$. A MVNS $A$ in $X$ is

$$
\left.A=\left\{x\left\langle T_{A}(x), I_{A}(x), F_{A}(x)\right\rangle \mid x \in X\right)\right\},
$$

where $T_{A}$ is the truth-membership function, $I_{A}$ is the indeterminacy-membership function, and $F_{A}$ is the falsity-membership function. $\gamma, \eta$ and $\xi$, respectively to represent any real values in $T_{A}, I_{A}$ and $F_{A}$, satisfying $0 \leq \gamma, \eta, \xi \leq 1 . \# T_{A}, \# I_{A}$ and $\# F_{A}$ are the numbers of all elements in $T_{A}, I_{A}$ and $F_{A}$.

If there is just one element in $X$, then $A$ can be called a MVNN, and $A$ is represented by $\left\langle T_{A}, I_{A}, F_{A}\right\rangle$; if $T_{A}, I_{A}$ and $F_{A}$ only have one value, then the MVNN will be reduced to a SVNN.

Definition $2[\mathbf{5 4}, \mathbf{5 5}]$. Let $\left.A=\left\{x\left\langle T_{A}(x), I_{A}(x), F_{A}(x)\right\rangle \mid x \in X\right)\right\}$ and $\left.B=\left\{x\left\langle T_{B}(x), I_{B}(x), F_{B}(x)\right\rangle \mid x \in X\right)\right\}$ be two MVNNs. Also let $\forall x \in X$, all values in $T_{A}(x), I_{A}(x)$ and $F_{A}(x)$ be ranked in ascending order. $\gamma^{i}, \eta^{i}$ and $\xi^{i}$ are the i-th value in $T(x), I(x)$ and $F(x)$. Then, we have: $A \leq B$ if $\gamma_{A}{ }^{k} \leq \gamma_{B}{ }^{k}, \gamma_{A}^{\# T} \leq \gamma_{B}^{\# T}$, $\eta_{A}^{l} \geq \eta_{B}^{l}, \quad \eta_{A}^{\# \mathrm{II}} \geq \eta_{B}^{\# I}, \quad \xi_{A}{ }^{m} \geq \xi_{B}{ }^{m} \quad$ and $\quad \xi_{A}{ }^{\# \mathrm{~F}} \geq \xi_{B}^{\# F}, \quad$ where $k=1,2, \cdots, \# T, \quad l=1,2, \cdots, \# I$, $m=1,2, \cdots, \# F$, and $\# T=\min \left(\# T_{A}(x), \# T_{B}(x)\right), \# I=\min \left(\# I_{A}(x), \# I_{B}(x)\right), \# F=\min \left(\# F_{A}(x), \# F_{B}(x)\right)$.

Definition 3. Let $A=\left\langle T_{A}, I_{A}, F_{A}\right\rangle$ and $B=\left\langle T_{B}, I_{B}, F_{B}\right\rangle$ be two MVNNs, and $\lambda>0$. The algebraic operations can be defined as follows: 
(1) $A \oplus B=\left(\begin{array}{c}\bigcup_{\gamma_{A} \in T_{A}, \gamma_{B} \in T_{B}}\left\{\gamma_{A}+\gamma_{B}-\gamma_{A} \gamma_{B}\right\}, \\ \bigcup_{\eta_{A} \in I_{A}, \eta_{B} \in I_{B}}\left\{\eta_{A} \eta_{B}\right\}, \\ \bigcup_{\xi_{A} \in F_{A}, \xi_{B} \in F_{B}}\left\{\xi_{A} \xi_{B}\right\}\end{array}\right)$;

(2) $A \otimes B=\left(\begin{array}{c}\bigcup_{\gamma_{A} \in T_{A}, \gamma_{B} \in T_{B}}\left\{\gamma_{A} \gamma_{B}\right\}, \\ \bigcup_{\eta_{A} \in I_{A}, \eta_{B} \in I_{B}}\left\{\eta_{A}+\eta_{B}-\eta_{A} \eta_{B}\right\}, \\ \bigcup_{\xi_{A} \in F_{A}, \xi_{B} \in F_{B}}\left\{\xi_{A}+\xi_{B}-\xi_{A} \xi_{B}\right\}\end{array}\right) ;$

(3) $\lambda A=\left(\begin{array}{c}\bigcup_{\gamma_{A} \in T_{A}} 1-\left(1-\gamma_{A}\right)^{\lambda} \\ \bigcup_{\eta_{A} \in I_{A}} \eta_{A}{ }^{\lambda} \\ \bigcup_{\xi_{A} \in F_{A}} \xi_{A}{ }^{\lambda}\end{array}\right)$;

(4) $A^{\lambda}=\left(\begin{array}{c}\bigcup_{\gamma_{A} \in T_{A}}\left\{\left(\gamma_{A}\right)^{\lambda}\right\}, \\ \bigcup_{\eta_{A} \in I_{A}}\left\{1-\left(1-\eta_{A}\right)^{\lambda}\right\} \\ \bigcup_{\xi_{A} \in F_{A}}\left\{1-\left(1-\xi_{A}\right)^{\lambda}\right\}\end{array}\right\rangle$.

\subsection{HM operators}

HM operators can tackle interrelationships among the aggregated arguments. In this subsection, two definitions of extending HM operators are introduced below.

Definition $4[56,57]$. Let $\theta=[0,1], s, t \geq 0, P^{s, t}: \theta^{n} \rightarrow \theta$, and then, the generalized Heronian mean operator is defined as follows:

$$
\operatorname{GHM}\left(z_{1}, z_{2}, \cdots, z_{m}\right)=\left(\frac{2}{m(m+1)} \sum_{h=1}^{m} \sum_{k=h}^{m} z_{h}^{s} z_{k}^{t}\right)^{\frac{1}{s+t}},
$$

Definition 5. Let $s, t \geq 0$, and $z_{h}(h=1,2, \cdots, m)$ be a set of nonnegative numbers. $Q=\left(q_{1}, q_{2}, \cdots, q_{m}\right)^{T}$ is the weight vector of $z_{h}(h=1,2, \cdots, m)$, and satisfies $q_{h}>0$ and $\sum_{h=1}^{m} q_{h}=1$. Then, IGWHM operator is defined below:

$$
\operatorname{IGWHM}^{s, t}\left(z_{1}, z_{2}, \cdots z_{n}\right)=\frac{\left(\sum_{h=1}^{m} \sum_{k=h}^{m} q_{h} q_{k} x_{h}^{s} x_{k}^{t}\right)^{\frac{1}{s+t}}}{\left(\sum_{h=1}^{m} \sum_{k=h}^{m} q_{h} q_{k}\right)^{\frac{1}{s+t}}} .
$$

\subsection{The MULTIMOORA method}

Let $A=\left\{A_{1}, A_{2}, \cdots, A_{m}\right\}$ be a collection of schemes, $C=\left\{C_{1}, C_{2}, \cdots, C_{n}\right\}$ be a set of criteria. $V=\left[v_{i j}\right]_{m \times n}$ 
represents original evaluation matrix, where $v_{i j}$ denotes attribute information for scheme $A_{i}$ under attribute $C_{j}$. In order to facilitate comparison, it is necessary to normalize $V$ and obtain normalized evaluation matrix $V^{*}=\left[v_{i j}^{*}\right]_{m \times n}$.

$$
v_{i j}{ }^{*}=\frac{v_{i j}}{\sqrt{\sum_{i=1}^{m} v_{i j}{ }^{2}}} .
$$

(1) The ratio system method

The comprehensive evaluation value of each scheme is derived from the following equation:

$$
y^{*}=\sum_{j=1}^{g} v_{i j}^{*}-\sum_{j=g+1}^{n} v_{i j}^{*}
$$

where $g$ and $n-g$ represents the number of benefit and cost criterion, respectively.

The best alternative can be obtained by the following formula:

$$
A^{*}=\left\{A_{i} \mid \max _{i} y_{i}^{*}\right\} \text {. }
$$

(2) The reference point method

Firstly, each optimal reference point of criterion is obtained below:

$$
r_{j}^{*}=\left\{\begin{array}{l}
\max _{i} v_{i j}{ }^{*}, j \leq g \\
\min _{i} v_{i j}{ }^{*}, j>g
\end{array} .\right.
$$

Then, comprehensive evaluation value of each scheme is derived from the following formula:

$$
z_{i}^{*}=\max _{j}\left|r_{j}^{*}-v_{i j}^{*}\right|
$$

where $\left|r_{j}^{*}-v_{i j}^{*}\right|$ represents deviation of each attribute value from reference point.

Finally, the best alternative can be obtained as follows:

$$
A^{*}=\left\{A_{i} \mid \min _{i} z_{i}^{*}\right\} \text {. }
$$

(3) The full multiplicative form method

The comprehensive evaluation value of each scheme is derived from the following equation:

$$
u_{i}^{*}=\frac{\prod_{j=1}^{g} v_{i j}{ }^{*}}{\prod_{j=g+1}^{n} v_{i j}{ }^{*}},
$$

where $\prod_{j=1}^{g} v_{i j}{ }^{*}$ represents the product of benefit criterion and $\prod_{j=g+1}^{n} v_{i j}{ }^{*}$ represents the product of cost criterion.

Then, the best alternative can be obtained as follows: 


$$
A^{*}=\left\{A_{i} \mid \max _{i} u_{i}^{*}\right\}
$$

\subsection{Prospect theory}

The prospect theory was developed by modifying the theory of maximum subjective expected utility [58]. The first phase of this theory is processing and reference point selection. The next phase is to judge and calculate information by value function and weight function. Such a decision-making process can reflect the limited rationality of the decision-maker.

The core of prospect theory is prospect value. It is expressed below:

$$
V=\sum_{i=1}^{n} \pi\left(p_{i}\right) v\left(\Delta x_{i}\right)
$$

while $\pi(p)$ represents the probability weight function considering risk attitude and $v\left(\Delta x_{i}\right)$ indicates the value function formed by the decision-maker's subjective feelings.

The probability weight function $\pi(p)$ and value function $v\left(\Delta x_{i}\right)$ are expressed as follows:

$$
\begin{gathered}
\pi(p)=\left\{\begin{array}{c}
\frac{p^{\gamma}}{\left(p^{\gamma}+\left(1-p^{\gamma}\right)\right)^{1 / \gamma}}, \Delta x \geq 0 \\
\frac{p^{\delta}}{\left(p^{\delta}+(1-p)^{\delta}\right)^{1 / \delta}}, \Delta x \leq 0
\end{array},\right. \\
v(x)=\left\{\begin{array}{c}
(\Delta x)^{\alpha}, \Delta x \geq 0 \\
-\lambda(\Delta x)^{\beta}, \Delta x \leq 0
\end{array}\right.
\end{gathered}
$$

while $\Delta x$ represents the difference between the decision criterion value and the reference point, $\alpha$ and $\beta$ represents risk attitude coefficients. $0 \leq \alpha, \beta \leq 1$, the greater the value of both of them, the more risk-taking decision-makers are. $\lambda$ represents loss avoidance coefficient.

\section{Methodology}

In this section, firstly, we propose the IGMVNWHM based on the IGWHM operator. Then, some properties about aggregation operator are presented. Secondly, a new distance measure between two MVNNs is defined. Finally, according to the prospect theory, an IMVN-PT-MULTIIMOORA method is presented for dealing with MCGDM problem.

\subsection{IGMVNWHM operator}

Definition 6. Let $s, t \geq 0$, and $\partial_{h}=\left\langle T_{h}, I_{h}, F_{h}\right\rangle(h=1,2, \cdots, m)$ be a set of MVNNs with the weights $Q=\left(q_{1}, q_{2}, \cdots q_{m}\right)^{T}$, satisfying $q_{h} \geq 0$ and $\sum q_{h}=1$, and then the IGMVNWHM operator is defined as 
follows:

$$
\operatorname{IGMVNWHM}^{s, t}\left(\partial_{1}, \partial_{2}, \cdots \partial_{m}\right)=\left(\frac{\bigoplus_{h=1}^{m} \bigoplus_{k=h}^{m}\left(q_{h} q_{k} \partial_{h}{ }^{s} \partial_{k}{ }^{t}\right)}{\sum_{h=1}^{m} \sum_{k=h}^{m} q_{h} q_{k}}\right)^{\frac{1}{s+t}},
$$

According to the algebraic operations in section 2.2, the following theorems can be obtained.

Theorem 1. Let $s, t \geq 0$, and $\partial_{h}=\left\langle T_{h}, I_{h}, F_{h}\right\rangle(h=1,2, \cdots, m)$ be a set of $m$ elements. $Q=\left(q_{1}, q_{2}, \cdots q_{m}\right)^{T}$ are the weights of all elements, satisfying $q_{h} \geq 0$ and $\sum q_{h}=1, \gamma_{A_{h}}, \eta_{A_{h}}$ and $\xi_{A_{h}}$, respectively representing all elements in $T_{A_{h}}, I_{A_{h}}$ and $F_{A_{h}}$; and with $\gamma_{B_{h}}, \eta_{B_{h}}$ and $\xi_{B_{h}}$ respectively representing all elements in $T_{B_{h}}, I_{B_{h}}$ and $F_{B_{h}}$. Then, the value aggregated by Eq. (14) is still an MVNN, and

$$
\begin{aligned}
& \operatorname{IGMVNWHM}^{s, t}\left(\partial_{1}, \partial_{2}, \cdots \partial_{m}\right)= \\
& \left(\begin{array}{c}
\bigcup_{\gamma_{h} \in T_{h}, \gamma_{k} \in T_{k}}\left\{\left(1-\left(\prod_{h=1}^{m} \prod_{k=h}^{m}\left(1-\gamma_{h}^{s} \gamma_{k}^{t}\right)^{q_{h} q_{k}}\right)^{\left.\left.\frac{1}{\sum_{h=1}^{m} \sum_{k=h}^{m} q_{h} q_{k}}\right)^{\frac{1}{s+t}}\right\},}\right.\right. \\
\bigcup_{\eta_{h} \in I_{h}, \eta_{k} \in I_{k}}\left\{1-\left(1-\left(\prod_{h=1}^{m} \prod_{k=h}^{m}\left(1-\left(1-\eta_{h}\right)^{s}\left(1-\eta_{k}\right)^{t}\right)^{q_{h} q_{k}}\right)^{\frac{1}{\sum_{h=1}^{m} \sum_{k=h}^{m} q_{h} q_{k}}}\right)^{\frac{1}{s+t}}\right\}, \\
\bigcup_{\xi_{h} \in F_{h}, \xi_{k} \in F_{k}}\left\{1-\left(1-\left(\prod_{h=1}^{m} \prod_{k=h}^{m}\left(1-\left(1-\xi_{h}\right)^{s}\left(1-\xi_{k}\right)^{t}\right)^{q_{h} q_{k}}\right)^{\frac{1}{\sum_{h=1}^{m} \sum_{k=h}^{m} q_{h} q_{k}}}\right)^{\frac{1}{s+t}}\right\}
\end{array}\right),
\end{aligned}
$$

Proof.

$$
\begin{aligned}
& \partial_{h}{ }^{s}=\left\langle\bigcup_{\gamma_{h} \in T_{h}}\left\{\gamma_{h}{ }^{s}\right\}, \bigcup_{\eta_{h} \in I_{h}}\left\{1-\left(1-\eta_{h}\right)^{s}\right\}, \bigcup_{\xi_{h} \in F_{h}}\left\{1-\left(1-\xi_{h}\right)^{s}\right\}\right\rangle, \\
& \partial_{k}{ }^{t}=\left\langle\bigcup_{\gamma_{k} \in T_{k}}\left\{\gamma_{k}{ }^{t}\right\}, \bigcup_{\eta_{k} \in I_{k}}\left\{1-\left(1-\eta_{k}\right)^{t}\right\}, \bigcup_{\xi_{k} \in F_{k}}\left\{1-\left(1-\xi_{k}\right)^{t}\right\}\right\rangle .
\end{aligned}
$$

Then

$$
q_{h} q_{k} \partial_{h}^{s} \otimes \partial_{k}{ }^{t}=\left(\begin{array}{l}
\bigcup_{\gamma_{h} \in T_{h}, \gamma_{k} \in T_{k}}\left\{1-\left(1-\gamma_{h}{ }^{s} \gamma_{k}^{t}\right)^{q_{h} q_{k}}\right\}, \\
\bigcup_{\eta_{h} \in I_{h}, \eta_{k} \in I_{k}}\left\{\left(1-\left(1-\eta_{h}\right)^{s}\left(1-\eta_{k}\right)^{t}\right)^{q_{h} q_{k}}\right\}, \\
\bigcup_{\xi_{h} \in F_{h}, \xi_{k} \in F_{k}}\left\{\left(1-\left(1-\xi_{h}\right)^{s}\left(1-\xi_{k}\right)^{t}\right)^{q_{h} q_{h}}\right\}
\end{array}\right\rangle .
$$

(1) If $m=2$, based on Eq. (14) and Eq. (16), we can determine that

$$
\begin{aligned}
& \operatorname{IGMVNWHM} M^{s, t}\left(\partial_{1}, \partial_{2}\right)=\left(\frac{\bigoplus_{h=1}^{2} \bigoplus_{k=h}^{2}\left(q_{h} q_{k} \partial_{h}{ }^{s} \partial_{k}{ }^{t}\right)}{\sum_{h=1}^{m} \sum_{k=h}^{m} q_{h} q_{k}}\right)^{\frac{1}{s+t}} \\
& =\left(\frac{q_{1} q_{1} \partial_{1}{ }^{s} \partial_{1}{ }^{t} \oplus q_{1} q_{2} \partial_{1}{ }^{s} \partial_{2}{ }^{t} \oplus q_{2} q_{2} \partial_{2}{ }^{s} \partial_{2}{ }^{t}}{\sum_{h=1}^{2} \sum_{k=h}^{2} q_{h} q_{k}}\right)^{\frac{1}{s+t}} \text {, }
\end{aligned}
$$


and

$$
\begin{aligned}
& q_{1} q_{1} \partial_{1}^{s} \partial_{1}^{t} \oplus q_{1} q_{2} \partial_{1}^{s} \partial_{2}{ }^{t} \oplus q_{2} q_{2} \partial_{2}^{s} \partial_{2}^{t} \\
& =\left(\begin{array}{l}
\bigcup_{\gamma_{1} \in T_{1}, \gamma_{2} \in T_{2}} 1-\left(1-\gamma_{1} s \gamma_{1}^{t}\right)^{q_{1} q_{1}}\left(1-\gamma_{1}^{s} \gamma_{2}^{t}\right)^{q_{1} q_{2}}\left(1-\gamma_{2}{ }^{s} \gamma_{2}^{t}\right)^{q_{2} q_{2}}, \\
\bigcup_{\eta_{1} \in I_{1}, \eta_{2} \in I_{2}}\left(1-\left(1-\eta_{1}\right)^{s}\left(1-\eta_{1}\right)^{t}\right)^{q_{1} q_{1}}\left(1-\left(1-\eta_{1}\right)^{s}\left(1-\eta_{2}\right)^{t}\right)^{q_{1} q_{2}}\left(1-\left(1-\eta_{2}\right)^{s}\left(1-\eta_{2}\right)^{t}\right)^{q_{2} q_{2}}, \\
\bigcup_{\xi_{1} \in F_{1}, \xi_{2} \in F_{2}}\left(1-\left(1-\xi_{1}\right)^{s}\left(1-\xi_{1}\right)^{t}\right)^{q_{1} q_{1}}\left(1-\left(1-\xi_{1}\right)^{s}\left(1-\xi_{2}\right)^{t}\right)^{q_{1} q_{2}}\left(1-\left(1-\xi_{2}\right)^{s}\left(1-\xi_{2}\right)^{t}\right)^{q_{2} q_{2}}
\end{array}\right)
\end{aligned}
$$

Then

$$
\left.\begin{array}{l}
\left(\begin{array}{c}
\left.\frac{q_{1} q_{1} \partial_{1}{ }^{s} \partial_{1}{ }^{t} \oplus q_{1} q_{2} \partial_{1}{ }^{s} \partial_{2}{ }^{t} \oplus q_{2} q_{2} \partial_{2}{ }^{s} \partial_{2}{ }^{t}}{\sum_{h=1}^{2} \sum_{k=h}^{2} q_{h} q_{k}}\right)^{\frac{1}{s+t}} \\
\bigcup_{\gamma_{1} \in T_{1}, \gamma_{2} \in T_{2}}\left\{\left(1-\left(\prod_{h=1}^{2} \prod_{k=h}^{2}\left(1-\gamma_{h}{ }^{s} \gamma_{k}{ }^{t}\right)^{q_{h} q_{k}}\right)^{\frac{1}{\sum_{h=1}^{m} \sum_{k=h}^{m} q_{h} q_{k}}}\right)^{\frac{1}{s+t}}\right\}, \\
\bigcup_{\eta_{1} \in I_{1}, \eta_{2} \in I_{2}}\left\{1-\left(1-\left(\prod_{h=1}^{2} \prod_{k=h}^{2}\left(1-\left(1-\eta_{h}\right)^{s}\left(1-\eta_{k}\right)^{t}\right)^{q_{h} q_{k}}\right)^{\left.\left.\frac{1}{\sum_{h=1}^{m} \sum_{k=h}^{m} q_{h} q_{k}}\right)^{\frac{1}{s+t}}\right\}}\right.\right. \\
\bigcup_{\xi_{1} \in F_{1}, \xi_{2} \in F_{2}}\left\{1-\left(1-\left(\prod_{h=1}^{2} \prod_{k=h}^{2}\left(1-\left(1-\xi_{h}\right)^{s}\left(1-\xi_{k}\right)^{t}\right)^{q_{h} q_{k}}\right)^{\frac{1}{\sum_{h=1}^{m} \sum_{k=h}^{m} q_{h} q_{k}}}\right)^{\frac{1}{s+t}}\right\}
\end{array}\right.
\end{array}\right) .
$$

(2) Assuming Eq. (15) holds for $m=g$, we can obtain

$$
\begin{aligned}
& \operatorname{IGMVNWHM}^{s, t}\left(\partial_{1}, \partial_{2}, \cdots \partial_{g}\right)=\left(\frac{1}{\sum_{h=1}^{g} \sum_{k=h}^{g} q_{h} q_{k}} \stackrel{g}{\oplus_{h=1}^{g}} \bigoplus_{k=h}^{g}\left(q_{h} q_{k} \partial_{h}{ }^{s} \otimes \partial_{k}{ }^{t}\right)\right)^{\frac{1}{s+t}} \\
& \left(\begin{array}{c}
\bigcup_{\gamma_{h} \in T_{h}, \gamma_{k} \in T_{k}}\left\{\left(1-\left(\prod_{h=1}^{g} \prod_{k=h}^{g}\left(1-\gamma_{h}^{s} \gamma_{k}^{t}\right)^{q_{h} q_{k}}\right)^{\left.\left.\frac{1}{\sum_{h=1}^{g} \sum_{k=h}^{g} q_{h} q_{k}}\right)^{\frac{1}{s+t}}\right\},}\right.\right. \\
\bigcup_{\eta_{h} \in I_{h}, \eta_{k} \in I_{k}}\left\{1-\left(1-\left(\prod_{h=1}^{g} \prod_{k=h}^{g}\left(1-\left(1-\eta_{h}\right)^{s}\left(1-\eta_{k}\right)^{t}\right)^{q_{h} q_{k}}\right)^{\left.\left.\frac{1}{\sum_{h=1}^{g} \sum_{k=h}^{g} q_{h} q_{k}}\right)^{\frac{1}{s+t}}\right\}}\right.\right. \\
\bigcup_{\xi_{h} \in F_{h}, \xi_{k} \in F_{k}}\left\{1-\left(1-\left(\prod_{h=1}^{g} \prod_{k=h}^{g}\left(1-\left(1-\xi_{h}\right)^{s}\left(1-\xi_{k}\right)^{t}\right)^{q_{h} q_{k}}\right)^{\frac{1}{\sum_{h=1}^{g} \sum_{k=h}^{g} q_{h} q_{k}}}\right)^{\frac{1}{s+t}}\right\}
\end{array}\right) .
\end{aligned}
$$

When $m=g+1$, according to Definition 3. Let $A=\left\langle T_{A}, I_{A}, F_{A}\right\rangle$ and $B=\left\langle T_{B}, I_{B}, F_{B}\right\rangle$ be two MVNNs, and $\lambda>0$. The algebraic operations can be defined as follows:

$$
\begin{aligned}
& \operatorname{IGMVNWHM}^{s, t}\left(\partial_{1}, \partial_{2}, \cdots \partial_{g}, \partial_{g+1}\right)=\left(\frac{1}{\sum_{h=1}^{g+1} \sum_{k=h}^{g+1} q_{h} q_{k}} \bigoplus_{h=1}^{g+1} \bigoplus_{k=h}^{g+1}\left(q_{h} q_{k} \partial_{h}{ }^{s} \otimes \partial_{k}{ }^{t}\right)\right)^{\frac{1}{s+t}} \\
& =\left(\frac{1}{\sum_{h=1}^{g+1} \sum_{k=h}^{g+1} q_{h} q_{k}}\left(\bigoplus_{h=1}^{g} \bigoplus_{k=h}^{g}\left(q_{h} q_{k} \partial_{h}{ }^{s} \otimes \partial_{k}{ }^{t}\right) \oplus \bigoplus_{h=1}^{g+1} q_{h} q_{g+1} \partial_{h}{ }^{s} \otimes \partial_{g+1}{ }^{t}\right)\right)^{\frac{1}{s+t}} .
\end{aligned}
$$

Then 


$$
\begin{aligned}
& \left.\bigoplus_{h=1}^{g} \bigoplus_{k=h}^{g}\left(q_{h} q_{k} \partial_{h}{ }^{s} \otimes \partial_{k}{ }^{t}\right) \oplus \oplus_{h=1}^{g+1} q_{h} q_{g+1} \partial_{h}{ }^{s} \otimes \partial_{g+1}{ }^{t}\right) \\
& =\left(\begin{array}{c}
\bigcup_{\gamma_{h} \in T_{h}, \gamma_{k} \in T_{k}}\left(1-\prod_{h=1}^{g} \prod_{k=h}^{g}\left(1-\gamma_{h}^{s} \gamma_{k}^{t}\right)^{q_{h} q_{k}} \prod_{h=1}^{g+1}\left(1-\gamma_{h}^{s} \gamma_{g+1}{ }^{t}\right)^{q_{h} q_{g+1}},\right. \\
\bigcup_{\eta_{h} \in I_{h}, \eta_{k} \in I_{k}} \prod_{h=1}^{g} \prod_{k=1}^{g}\left(1-\left(1-\eta_{h}\right)^{s}\left(1-\eta_{k}\right)^{t}\right)^{q_{h} q_{k}} \prod_{h}^{g+1}\left(1-\left(1-\eta_{h}\right)^{s}\left(1-\eta_{g+1}\right)^{t}\right)^{q_{h} q_{g+1}}, \\
\bigcup_{\xi_{h} \in F_{h}, \xi_{k} \in F_{k}} \prod_{h=1}^{g} \prod_{k=1}^{g}\left(1-\left(1-\xi_{h}\right)^{s}\left(1-\xi_{k}\right)^{t}\right)^{q_{h} q_{k}} \prod_{h}^{g+1}\left(1-\left(1-\xi_{h}\right)^{s}\left(1-\xi_{g+1}\right)^{t}\right)^{q_{h} q_{g+1}}
\end{array}\right\rangle .
\end{aligned}
$$

Further

$$
\left.\begin{array}{l}
\operatorname{IGMVNWHM} M^{s, t}\left(\partial_{1}, \partial_{2}, \cdots \partial_{g+1}\right) \\
\bigcup_{\gamma_{h} \in T_{h}, \gamma_{k} \in T_{k}}\left\{\left(1-\left(\prod_{h=1}^{g+1} \prod_{k=i}^{g+1}\left(1-\gamma_{h}^{s} \gamma_{k}^{t}\right)^{q_{h} q_{k}}\right)^{\left.\left.\frac{1}{\sum_{h=1}^{g+1} \sum_{k=h}^{g+1} q_{h} q_{k}}\right)^{\frac{1}{s+t}}\right\},}\right.\right. \\
\bigcup_{\eta_{h} \in I_{h}, \eta_{k} \in I_{k}}\left\{1-\left(1-\left(\prod_{h=1}^{g+1} \prod_{k=h}^{g+1}\left(1-\left(1-\eta_{h}\right)^{s}\left(1-\eta_{k}\right)^{t}\right)^{q_{h} q_{k}}\right)^{\left.\left.\frac{1}{\sum_{h=1}^{s+1} \sum_{k=h}^{g+1} q_{h} q_{k}}\right)^{\frac{1}{s+t}}\right\}}\right.\right. \\
\bigcup_{\xi_{h} \in F_{h}, \xi_{k} \in F_{k}}\left\{1-\left(1-\left(\prod_{h=1}^{g+1} \prod_{k=h}^{g+1}\left(1-\left(1-\xi_{h}\right)^{s}\left(1-\xi_{k}\right)^{t}\right)^{q_{h} q_{k}}\right)^{\left.\left.\frac{1}{\sum_{h=1}^{g+1} \sum_{k=h}^{g+1} q_{h} q_{k}}\right)^{\frac{1}{s+t}}\right\}}\right.\right.
\end{array}\right) .
$$

i.e., Eq. (15) holds for $m=g+1$. Thus, Eq. (15) holds for all $m$.

Therefore

$$
\begin{aligned}
& \operatorname{IGMVNWHM}{ }^{s, t}\left(\partial_{1}, \partial_{2}, \cdots \partial_{m}\right)=\left(\frac{1}{\sum_{h=1}^{m} \sum_{k=h}^{m} q_{h} q_{k}} \bigoplus_{h=1}^{m} \oplus_{k=h}^{m}\left(q_{h} q_{k} \partial_{h}{ }^{s} \otimes \partial_{k}{ }^{t}\right)\right)^{\frac{1}{s+t}} \\
& \left(\begin{array}{c}
\bigcup_{\gamma_{h} \in T_{h}, \gamma_{k} \in T_{k}}\left\{\left(1-\left(\prod_{h=1}^{m} \prod_{k=h}^{m}\left(1-\gamma_{h}^{s} \gamma_{k}^{t}\right)^{q_{h} q_{k}}\right)^{\left.\left.\frac{1}{\sum_{h=1}^{m} \sum_{k=h}^{m} q_{h} q_{k}}\right)^{\frac{1}{s+t}}\right\},}\right.\right. \\
\bigcup_{\eta_{h} \in I_{h}, \eta_{k} \in I_{k}}\left\{1-\left(1-\left(\prod_{h=1}^{m} \prod_{k=h}^{m}\left(1-\left(1-\eta_{h}\right)^{s}\left(1-\eta_{k}\right)^{t}\right)^{q_{h} q_{k}}\right)^{\frac{1}{\sum_{h=1}^{m} \sum_{k=h}^{m} q_{h} q_{k}}}\right)^{\frac{1}{s+t}}\right\} \\
\bigcup_{\xi_{h} \in F_{h}, \xi_{k} \in F_{k}}\left\{1-\left(1-\left(\prod_{h=1}^{m} \prod_{k=h}^{m}\left(1-\left(1-\xi_{h}\right)^{s}\left(1-\xi_{k}\right)^{t}\right)^{q_{h} q_{k}}\right)^{\left.\left.\frac{1}{\sum_{h=1}^{m} \sum_{k=h}^{m} q_{h} q_{k}}\right)^{\frac{1}{s+t}}\right\}}\right.\right.
\end{array}\right) .
\end{aligned}
$$

In addition, there are some properties of IGMVNWHM operators.

Theorem 2 (Monotonicity). Let $A_{h}=\left\langle T_{A_{h}}, I_{A_{h}}, F_{A_{h}}\right\rangle$ and $B_{h}=\left\langle T_{B_{h}}, I_{B_{h}}, F_{B_{h}}\right\rangle(h=1,2, \cdots, m)$ be two sets of MVNNs. If $A_{h} \leq B_{h}$ for all $h, \gamma_{A_{h}} \leq \gamma_{B_{h}}, \eta_{A_{h}} \geq \eta_{B_{h}}$ and $\xi_{A_{h}} \geq \xi_{B_{h}}$, then

$$
\operatorname{IGMVNWHM}^{s, t}\left(A_{1}, A_{2}, \cdots A_{m}\right) \leq \operatorname{IGMVNWHM}^{s, t}\left(B_{1}, B_{2}, \cdots B_{m}\right),
$$

where $\gamma_{A_{h}}, \eta_{A_{h}}$ and $\xi_{A_{h}}$ respectively represent all elements in $T_{A_{h}}, I_{A_{h}}$ and $F_{A_{h}} ; \gamma_{B_{h}}, \eta_{B_{h}}$ and $\xi_{B_{h}}$ 
respectively represent all elements in $T_{B_{h}}, I_{B_{h}}$ and $F_{B_{h}}$.

Proof

Since $\gamma_{A_{h}} \leq \gamma_{B_{h}}$ for all $h$, and $s, t \geq 0$, we have

$$
\begin{aligned}
& \gamma_{A_{h}}{ }^{s} \cdot \gamma_{A_{k}}{ }^{t} \leq \gamma_{B_{h}}{ }^{s} \cdot \gamma_{B_{k}}{ }^{t}, \quad 1-\gamma_{A_{h}}{ }^{s} \cdot \gamma_{A_{k}}{ }^{t} \geq 1-\gamma_{B_{h}}{ }^{s} \cdot \gamma_{B_{k}}{ }^{t} ; \\
& \prod_{h=1}^{m} \prod_{k=h}^{m}\left(1-\gamma_{A_{h}}{ }^{s} \cdot \gamma_{A_{k}}{ }^{t}\right)^{q_{h} q_{k}} \geq \prod_{h=1}^{m} \prod_{k=h}^{m}\left(1-\gamma_{B_{h}}{ }^{s} \cdot \gamma_{B_{k}}{ }^{t}\right)^{q_{h} q_{k}} .
\end{aligned}
$$

Therefore

$$
\left(1-\left(\prod_{h=1}^{m} \prod_{k=h}^{m}\left(1-\gamma_{A_{h}}{ }^{s} \cdot \gamma_{A_{k}}{ }^{t}\right)^{q_{h} q_{k}}\right)^{\frac{1}{\sum_{h=1}^{m} \sum_{k=h}^{m} q_{h} q_{k}}}\right)^{\frac{1}{s+t}} \leq\left(1-\left(\prod_{h=1}^{m} \prod_{k=h}^{m}\left(1-\gamma_{B_{h}}{ }^{s} \cdot \gamma_{B_{k}}{ }^{t}\right)^{q_{h} q_{k}}\right)^{\frac{1}{\sum_{h=1}^{m} \sum_{k=h}^{m} q_{h} q_{k}}}\right)^{\frac{1}{s+t}} .
$$

(1) Since $\eta_{A_{h}} \geq \eta_{B_{h}}$ for all $h$, and $s, t \geq 0$, we can derive

$$
\left(1-\eta_{h}\right)^{s} \geq\left(1-\eta_{B_{h}}\right)^{s},\left(1-\eta_{A_{k}}\right)^{t} \geq\left(1-\eta_{B_{k}}\right)^{t}
$$

And

$$
\begin{gathered}
1-\left(1-\eta_{A_{h}}\right)^{s}\left(1-\eta_{A_{k}}\right)^{t} \leq 1-\left(1-\eta_{B_{h}}\right)^{s}\left(1-\eta_{B_{k}}\right)^{t} ; \\
\prod_{h=1}^{m} \prod_{k=h}^{m}\left(1-\left(1-\eta_{A_{h}}\right)^{s}\left(1-\eta_{A_{k}}\right)^{t}\right)^{q_{h} q_{k}} \leq \prod_{h=1}^{m} \prod_{k=h}^{m}\left(1-\left(1-\eta_{B_{h}}\right)^{s}\left(1-\eta_{B_{k}}\right)^{t}\right)^{q_{h} q_{k}} .
\end{gathered}
$$

Therefore

$$
1-\left(1-\left(\prod_{h=1}^{m} \prod_{k=h}^{m}\left(1-\left(1-\eta_{A_{h}}\right)^{s}\left(1-\eta_{A_{k}}\right)^{t}\right)^{q_{h} q_{k}}\right)^{\frac{1}{\sum_{k=1}^{m} \sum_{k=h}^{m} q_{h} q_{k}}}\right)^{\frac{1}{s+t}} \leq 1-\left(1-\left(\prod_{h=1}^{m} \prod_{k=h}^{m}\left(1-\left(1-\eta_{B_{h}}\right)^{s}\left(1-\eta_{B_{k}}\right)^{t}\right)^{q_{h} q_{k}}\right)^{\left.\frac{1}{\sum_{h=1}^{m} \sum_{k=h}^{m} q_{h} q_{k}}\right)^{\frac{1}{s+t}}} .\right.
$$

(2) According to (1), it is not difficult to prove

$1-\left(1-\left(\prod_{h=1}^{m} \prod_{k=h}^{m}\left(1-\left(1-\xi_{A_{h}}\right)^{s}\left(1-\xi_{A_{k}}\right)^{t}\right)^{q_{h} q_{k}}\right)^{\frac{1}{\sum_{h=1}^{m} \sum_{k=h}^{m} q_{h} q_{k}}}\right)^{\frac{1}{s+t}} \leq 1-\left(1-\left(\prod_{h=1}^{m} \prod_{k=h}^{m}\left(1-\left(1-\xi_{B_{h}}\right)^{s}\left(1-\xi_{B_{k}}\right)^{t}\right)^{q_{h} q_{k}}\right)^{\frac{1}{\sum_{h=1}^{m} \sum_{k=h}^{m} q_{h} q_{k}}} \frac{1}{)^{s+t}}\right.$.

Based on (1)-(2), we can obtain

11 


$$
\begin{aligned}
& \left|\begin{array}{c}
\bigcup_{\gamma_{A_{h}} \in T_{A_{h}}, \gamma_{A_{k}} \in T_{A_{k}}}\left(1-\left(\prod_{h=1}^{m} \prod_{k=h}^{m}\left(1-\gamma_{A_{h}}{ }^{s} \cdot \gamma_{A_{k}}{ }^{t}\right)^{q_{h} q_{k}}\right)^{\left.\frac{1}{\sum_{h=1}^{m} \sum_{k=h}^{m} q_{h} q_{k}}\right)^{\frac{1}{s+t}}}\right. \\
\bigcup_{\eta_{A_{h}} \in I_{A_{h}}, \eta_{A_{k}} \in I_{A_{k}}} 1-\left(1-\left(\prod_{h=1}^{m} \prod_{k=h}^{m}\left(1-\left(1-\eta_{A_{h}}\right)^{s}\left(1-\eta_{A_{k}}\right)^{t}\right)^{q_{h} q_{k}}\right)^{\left.\frac{1}{\sum_{h=1}^{m} \sum_{k=h}^{m} q_{h} q_{k}}\right)^{\frac{1}{s+t}}}\right. \\
\bigcup_{\xi_{A_{h}} \in F_{A_{h}}, \xi_{A_{k}} \in F_{A_{k}}} 1-\left(1-\left(\prod_{h=1}^{m} \prod_{k=h}^{m}\left(1-\left(1-\xi_{A_{h}}\right)^{s}\left(1-\xi_{A_{k}}\right)^{t}\right)^{q_{h} q_{k}}\right)^{\frac{1}{\sum_{h=1}^{m} \sum_{k=h}^{m} q_{h} q_{k}}}\right)^{\frac{1}{s+t}}
\end{array}\right\rangle \leq \\
& \left\{\begin{array}{c}
\bigcup_{\gamma_{B_{h}} \in T_{B_{h}}, \gamma_{B_{k}} \in T_{B_{k}}}\left(1-\left(\prod_{h=1}^{m} \prod_{k=h}^{m}\left(1-\gamma_{B_{h}}{ }^{s} \cdot \gamma_{B_{k}}{ }^{t}\right)^{q_{h} q_{k}}\right)^{\left.\frac{1}{\sum_{h=1}^{m} \sum_{k=h}^{m} q_{h} q_{k}}\right)^{\frac{1}{s+t}}}\right. \\
\bigcup_{\eta_{B_{h}} \in I_{B_{h}}, \eta_{B_{k}} \in I_{B_{k}}} 1-\left(1-\left(\prod_{h=1}^{m} \prod_{k=h}^{m}\left(1-\left(1-\eta_{B_{h}}\right)^{s}\left(1-\eta_{B_{k}}\right)^{t}\right)^{q_{h} q_{k}}\right)^{\left.\frac{1}{\sum_{h=1}^{m} \sum_{k=h}^{m} q_{h} q_{k}}\right)^{\frac{1}{s+t}}}\right. \\
\bigcup_{\xi_{B_{h}} \in F_{B_{h}}, \xi_{B_{k}} \in F_{B_{k}}} 1-\left(1-\left(\prod_{h=1}^{m} \prod_{k=h}^{m}\left(1-\left(1-\xi_{B_{h}}\right)^{s}\left(1-\xi_{B_{k}}\right)^{t}\right)^{q_{h} q_{k}}\right)^{\left.\frac{1}{\sum_{h=1}^{m} \sum_{k=h}^{m} q_{h} q_{k}}\right)^{\frac{1}{s+t}}}\right.
\end{array}\right\rangle
\end{aligned}
$$

i.e., $\operatorname{IGMVNWHM}^{s, t}\left(A_{1}, A_{2}, \cdots A_{m}\right) \leq \operatorname{IGMVNWHM}^{s, t}\left(B_{1}, B_{2}, \cdots B_{m}\right)$.

Theorem 3 (Idempotency). Let $\partial_{h}=\left\langle T_{h}, I_{h}, F_{h}\right\rangle(h=1,2, \cdots, m)$ be a set of MVNNs, and $\partial=\langle T, I, F\rangle$. If $\gamma_{h}=\gamma, \eta_{h}=\eta$ and $\xi_{h}=\xi$ for all $h$, then

$$
\operatorname{IGMVNWHM}^{s, t}\left(\partial_{1}, \partial_{2}, \cdots, \partial_{m}\right)=\partial,
$$

where $\gamma_{h}, \eta_{h}, \xi_{h}$ and $\gamma, \eta, \xi$ respectively represent all the elements in $T_{h}, I_{h}, F_{h}$ and $T, I, F$.

Proof

Since $\partial_{h}=(T, I, F)(h=1,2, \cdots m)$, and based on Eq. (15), we can derive

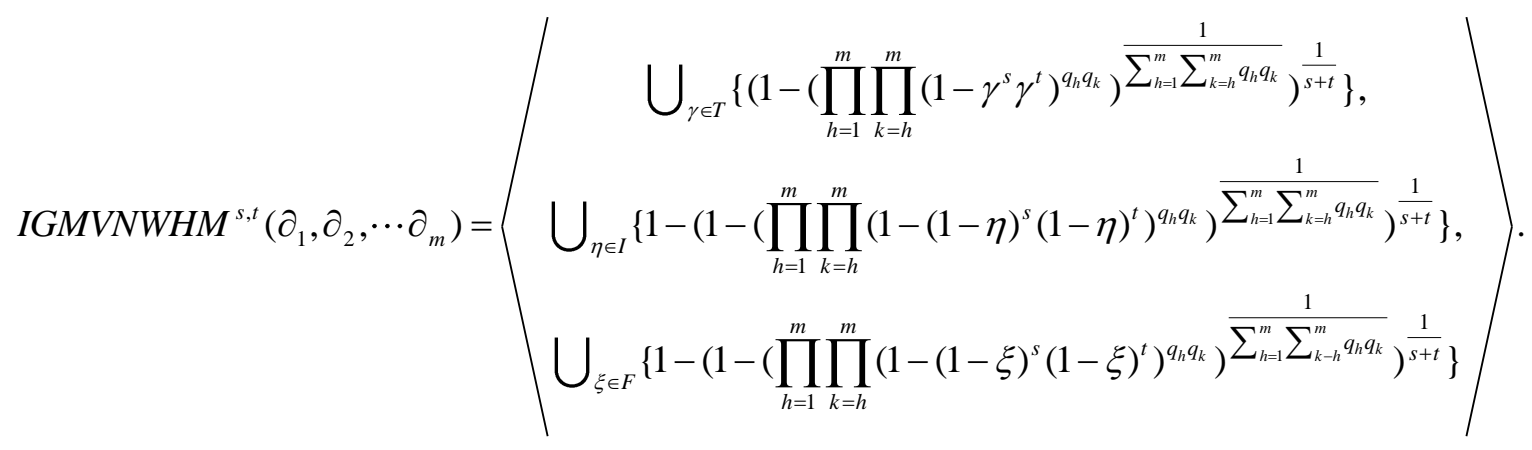

Then 


$$
=\left\langle\begin{array}{c}
\bigcup_{\gamma \in T}\left(1-\left(1-\gamma^{s+t}\right)\right)^{\frac{1}{s+t}}, \\
\bigcup_{\eta \in I} 1-\left(1-\left(1-(1-\eta)^{s+t}\right)\right)^{\frac{1}{s+t}}, \\
\bigcup_{\xi \in F} 1-\left(1-\left(1-(1-\xi)^{s+t}\right)\right)^{\frac{1}{s+t}}
\end{array}\right\rangle=\left\langle\begin{array}{c}
\bigcup_{\gamma \in T} \gamma \\
\bigcup_{\eta \in I} \eta \\
\bigcup_{\xi \in F} \xi
\end{array}\right\rangle=\langle T, I, F\rangle .
$$

\subsection{Distance measure between two MVNNs}

Definition 7. Let $C=\left\langle T_{C}, I_{C}, F_{C}\right\rangle$ and $E=\left(T_{E}, I_{E}, F_{E}\right)$ be two MVNNs, then the distance between $C$ and $E$ can be obtained by the following equation:

$$
d_{g d}(C, E)=\left(\frac{1}{\frac{1}{6}}\left[\begin{array}{l}
\frac{1}{\# T_{C}} \sum_{\gamma_{C} \in T_{C}} \min _{\gamma_{E} \in T_{E}}\left|\gamma_{C}-\gamma_{E}\right|^{\mu}+\frac{1}{\# T_{E}} \sum_{\gamma_{E} \in T_{E}} \min _{\gamma_{C} \in T_{C}}\left|\gamma_{E}-\gamma_{C}\right|^{\mu}+\frac{1}{\# I_{C}} \sum_{\eta_{C} \in I_{C}} \min _{\eta_{E} \in I_{E}}\left|\eta_{C}-\eta_{E}\right|^{\mu} \\
+\frac{1}{\# I_{E}} \sum_{\eta_{E} \in I_{E}} \min _{\eta_{C} \in I_{C}}\left|\eta_{E}-\eta_{C}\right|^{\mu}+\frac{1}{\# F_{C}} \sum_{\xi_{C} \in F_{C}} \min _{\xi_{E} \in F_{E}}\left|\xi_{C}-\xi_{E}\right|^{\mu}+\frac{1}{\# F_{E}} \sum_{\xi_{E} \in F_{E}} \min _{\xi_{C} \in F_{C}}\left|\xi_{E}-\xi_{C}\right|^{\mu}
\end{array}\right]\right)^{\frac{1}{\mu}}
$$

Theorem 4. Let $C=\left\langle T_{C}, I_{C}, F_{C}\right\rangle, D=\left(T_{D}, I_{D}, F_{D}\right)$ and $E=\left(T_{E}, I_{E}, F_{E}\right)$ be three MVNNs. The distance measure in Definition 7 satisfies the following properties:

(1) $d_{g d}(C, C)=0$;

(2) $d_{g d}(C, D)=d_{g d}(D, C)$;

(3) If $C \leq D \leq E$, then $d_{g d}(C, D) \leq d_{g d}(C, E)$ and $d_{g d}(D, E) \leq d_{g d}(C, E)$.

Proof.

Clearly, the distance measure satisfies properties (1) and (2). The proof of property (3) is shown below.

Since $C \leq D \leq E$, then by Definition 2, we can obtain that $\gamma_{C}{ }^{k} \leq \gamma_{D}{ }^{k} \leq \gamma_{E}{ }^{k}, \gamma_{C}^{\# T} \leq \gamma_{D}^{\# T} \leq \gamma_{E}^{\# T}$, $\eta_{C}{ }^{l} \geq \eta_{D}{ }^{l} \geq \eta_{E}^{l}, \quad \eta_{C}{ }^{\# \mathrm{I}} \geq \eta_{D}^{\# I} \geq \eta_{E}^{\# I}, \quad \xi_{C}{ }^{m} \geq \xi_{D}{ }^{m} \geq \xi_{E}{ }^{m} \quad$ and $\quad \xi_{C}^{\# F} \geq \xi_{D}^{\# F} \geq \xi_{E}^{\# F}$, where $k=1,2, \cdots, \# T$, $l=1,2, \cdots, \# I \quad, \quad m=1,2, \cdots, \# F \quad, \quad$ and $\quad \# T=\min \left(\# T_{C}(x), \# T_{D}(x), \# T_{E}(x)\right) \quad$, $\# I=\min \left(\# I_{C}(x), \# I_{D}(x), \# I_{E}(x)\right), \quad \# F=\min \left(\# F_{C}(x), \# F_{D}(x), \# F_{E}(x)\right)$. Subsequently, the following inequalities can be obtained.

$$
\left|\gamma_{C}{ }^{k}-\gamma_{D}{ }^{k}\right| \leq\left|\gamma_{C}{ }^{k}-\gamma_{F}{ }^{k}\right|,\left|\eta_{C}{ }^{l}-\eta_{D}{ }^{l}\right| \leq\left|\eta_{C}{ }^{l}-\eta_{F}{ }^{l}\right|, \quad\left|\xi_{C}{ }^{m}-\xi_{D}{ }^{m}\right| \leq\left|\xi_{C}{ }^{m}-\xi_{F}{ }^{m}\right|
$$

and

$$
\left|\gamma_{D}{ }^{k}-\gamma_{C}{ }^{k}\right| \leq\left|\gamma_{E}{ }^{k}-\gamma_{C}{ }^{k}\right|,\left|\eta_{D}{ }^{l}-\eta_{C}{ }^{l}\right| \leq\left|\eta_{E}{ }^{l}-\eta_{C}{ }^{l}\right|,\left|\xi_{D}{ }^{m}-\xi_{C}{ }^{m}\right| \leq\left|\xi_{E}{ }^{m}-\xi_{C}{ }^{m}\right| .
$$


Then,

$$
\begin{aligned}
& \frac{1}{\# T_{C}} \sum_{\gamma_{C} \in T_{C}} \min _{\gamma_{D} \in T_{D}}\left|\gamma_{C}-\gamma_{D}\right|^{\mu} \leq \frac{1}{\# T_{C}} \sum_{\gamma_{C} \in T_{C}} \min _{\gamma_{E} \in T_{E}}\left|\gamma_{C}-\gamma_{E}\right|^{\mu}, \\
& \frac{1}{\# I_{C}} \sum_{\eta_{C} \in I_{C}} \min _{\eta_{D} \in I_{D}}\left|\eta_{C}-\eta_{D}\right|^{\mu} \leq \frac{1}{\# I_{C}} \sum_{\eta_{C} \in I_{C}} \min _{\eta_{E} \in I_{E}}\left|\eta_{C}-\eta_{E}\right|^{\mu}, \\
& \frac{1}{\# F_{C}} \sum_{\xi_{C} \in F_{C}} \min _{\xi_{D} \in F_{D}}\left|\xi_{C}-\xi_{D}\right|^{\mu} \leq \frac{1}{\# F_{C}} \sum_{\xi_{C} \in F_{C}} \min _{\xi_{E} \in F_{E}}\left|\xi_{C}-\xi_{E}\right|^{\mu}, \\
& \frac{1}{\# T_{D}} \sum_{\gamma_{D} \in T_{D}} \min _{\gamma_{C} \in T_{C}}\left|\gamma_{D}-\gamma_{C}\right|^{\mu} \leq \frac{1}{\# T_{E}} \sum_{\gamma_{E} \in T_{E}} \min _{\gamma_{C} \in T_{C}}\left|\gamma_{E}-\gamma_{C}\right|^{\mu}, \\
& \frac{1}{\# I_{D}} \sum_{\eta_{D} \in I_{D}} \min _{\eta_{C} \in I_{C}}\left|\eta_{D}-\eta_{C}\right|^{\mu} \leq \frac{1}{\# I_{E}} \sum_{\eta_{E} \in I_{E}} \min _{\eta_{C} \in I_{C}}\left|\eta_{E}-\eta_{C}\right|^{\mu}, \\
& \frac{1}{\# F_{D}} \sum_{\xi_{D} \in F_{D}} \min _{\xi_{C} \in F_{C}}\left|\xi_{D}-\xi_{C}\right|^{\mu} \leq \frac{1}{\# F_{E}} \sum_{\xi_{E} \in F_{E}} \min _{\xi_{C} \in F_{C}}\left|\xi_{E}-\xi_{C}\right|^{\mu} .
\end{aligned}
$$

Thus, $d_{g d}(C, D) \leq d_{g d}(C, E)$. Similarly, we can also get $d_{g d}(D, E) \leq d_{g d}(C, E)$.

\subsection{IMVN-PT-MULTIMOORA method}

The traditional MULTIMOORA method assumes that decision-makers are entirely rational, which is inconsistent with the actual condition. Therefore, it is necessary to use relevant theories to solve this problem. In this section, we connect the prospect theory with MULTIMOORA method. In addition, a new weights determination method is applied into the multi-valued neutrosophic MULTIMOORA method.

Let $A=\left\{A_{1}, A_{2}, \cdots, A_{m}\right\}$ be a collection of alternatives, $C=\left\{C_{1}, C_{2}, \cdots, C_{n}\right\}$ be a set of criteria. Assume that $Q=\left\{q_{1}, q_{2}, \cdots, q_{n}\right\} \quad\left(q_{k} \in[0,1]\right.$ and $\left.\sum_{k=1}^{n} q_{k}=1\right)$ is weight. The decision matrix is $B=\left[b_{h k}\right]_{m \times n}$, where $b_{h k}$ is a MVNN that represents the assessment of alternative $A_{h}(h=1,2, \cdots, m)$ with the criterion $C_{k}$ $(k=1,2, \ldots, n)$. The standardization is calculated below:

$$
\tilde{b_{h k}}=\left\{\begin{array}{c}
\frac{p^{\gamma}}{\left(p^{\gamma}+(1-p)^{\gamma}\right)^{1 / \gamma}}\left(d\left(b_{h k}, \tilde{b_{k}}\right)\right)^{\alpha}, b_{h k}>\tilde{b_{k}} \\
0, b_{h k}=\tilde{b_{k}} \\
-\lambda \frac{p^{\delta}}{\left(p^{\delta}+(1-p)^{\delta}\right)^{1 / \delta}}\left(d\left(b_{h k}, \tilde{b_{k}}\right)\right)^{\beta}, b_{h k}<\tilde{b_{k}}
\end{array},\right.
$$




$$
b_{h k}^{*}=\frac{\tilde{b_{h k}}}{\sqrt{\sum_{h=1}^{m}\left(\tilde{b_{h k}}\right)^{2}}},
$$

where $\tilde{b_{h k}}$ denotes the prospect value, $\tilde{b_{k}}$ denotes the reference point value of criterion $C_{k}, d\left(b_{h k}, \tilde{b_{k}}\right)$ denotes the distance between evaluation value and reference point, $b_{h k}{ }^{*}$ is the standardized form of $\tilde{b_{h k}}$, $B^{*}=\left[b_{h k}{ }^{*}\right]$ is the standardized decision matrix.

Before we conduct the MULTIMOORA method, the weights of criteria should be obtained. Firstly, for each criterion, the optimistic and pessimistic evaluation values are represented as follows: Optimistic values: $B^{+}=\left(b_{1}^{+}, b_{2}^{+}, \cdots, b_{n}^{+}\right)$and pessimistic values: $B^{-}=\left(b_{1}^{-}, b_{2}^{-}, \cdots, b_{n}^{-}\right)$. Then, according to the distance measure represented in Definition 7, we can get the distances between evaluation value of each criterion and optimistic/pessimistic values.

$$
d_{k}^{+}=\sum_{h=1}^{m} d\left(b_{h k}, b_{k}^{+}\right), d_{j}^{-}=\sum_{h=1}^{m} d\left(b_{h k}, b_{k}^{-}\right)
$$

Based on the TOPSIS method, the dispersion measure of criterion $C_{k}$ can be obtained below:

$$
e_{k}=\frac{d_{k}^{+}}{d_{k}^{+}+d_{k}^{-}} \text {. }
$$

Finally, the criterion weight can be obtained according to dispersion measure.

$$
q_{k}=\frac{e_{k}}{\sum_{k=1}^{n} e_{k}} .
$$

(1) The improved multi-valued neutrosophic- prospect theory (IMVN-PT)-ratio system method

The comprehensive evaluation value of each scheme is derived from the following equation:

$$
y_{h}^{*}=\sum_{k=1}^{g} q_{k} b_{h k}^{*}-\sum_{k=g+1}^{n} q_{k} b_{h k}^{*}
$$

where $g$ and $n-g$ represents the number of benefit and cost criterion, respectively.

The best alternative can be obtained by the following formula:

$$
A_{R S}^{*}=\left\{A_{h} \mid \max _{h} y_{h}^{*}\right\} .
$$

(2) The IMVN-PT-reference point method

Firstly, each optimal reference point of criterion is obtained below:

$$
r_{k}^{*}=\left\{\begin{array}{l}
\max _{h} b_{h k}{ }^{*}, k \leq g \\
\min _{h} b_{h k}{ }^{*}, k>g
\end{array} .\right.
$$


Then, comprehensive evaluation value of each scheme is derived from the following formula:

$$
z_{h}^{*}=\max _{k} q_{k}\left|r_{k}^{*}-b_{h k}^{*}\right|,
$$

Finally, the best alternative can be obtained as follows:

$$
A_{R P}^{*}=\left\{A_{h} \mid \min _{h} z_{h}^{*}\right\} .
$$

(3) The IMVN-PT-full multiplicative form method

The comprehensive evaluation value of $A_{h}$ can be obtained as follows:

$$
u_{h}{ }^{*}=\frac{\prod_{k=1}^{g}\left(b_{h k}{ }^{*}\right)^{q_{k}}}{\prod_{k=g+1}^{n}\left(b_{h k}{ }^{q_{k}}\right.} .
$$

The best alternative can be obtained as follows:

$$
A_{F M F}^{*}=\left\{A_{h} \mid \max _{h} u_{h}^{*}\right\} .
$$

Based on the dominance theory, the final ranks can be collected from the above three parts of MULTIMOORA method.

\section{Solution framework for MVN-MCGDM problem}

Considering that the evaluation information are described by MVNNs, let $A=\left\{A_{1}, A_{2}, \cdots, A_{m}\right\}$ be the set of schemes, $D=\left\{D_{1}, D_{2}, \cdots, D_{l}\right\}$ be a set of decision-makers, the weights of decision-makers are expressed by $\phi=\left(\phi_{1}, \phi_{2}, \cdots, \phi_{l}\right), \phi_{k} \in[0,1]$ and $\sum_{k=1}^{l} \phi_{k}=1$. The criteria are expressed by $C=\left\{C_{1}, C_{2}, \cdots, C_{n}\right\}$. $Q=\left(q_{1}, q_{2}, \cdots, q_{n}\right)$ represents the importance of the criteria, satisfying $q_{k} \in[0,1](k=1,2, \cdots, n)$ and $\sum q_{k}=1$. We assume that the decision matrix is $B^{r}=\left[b_{h k}{ }^{r}\right]_{m \times n}$, where $b_{h k}{ }^{r}=\left\langle T_{h k}, I_{h k}, F_{h k}\right\rangle$ is a MVNN that represents the assessment of scheme $A_{h}(h=1,2, \ldots, m)$ under the criterion $C_{j}(k=1,2, \ldots, n)$ obtained from decision-maker $D_{r}$.

The solution framework is shown in Figure 1 which is at the end of this paper, and the detailed steps are stated below:

Step 1: Tidy up original data.

Gather and transform the evaluation information into MVNNs, then, normalize the decision matrix of each decision-maker based on following equation. 


$$
b_{h k}=\left\{\begin{array}{lr}
\partial_{h k}, & \text { if } C_{k} \text { is a benefit criteria } \\
\partial_{h k}, & \text { else }
\end{array},\right.
$$

where $\partial_{h k}{ }^{c}$ is the complement of $\partial_{h k}$, satisfying $\partial_{h k}^{c}=\left\langle F_{h k}, 1-I_{h k}, T_{h k}\right\rangle$.

Step 2: Obtain the collective decision matrix by IGMVNWHM operator.

According to Eq. (14), we can aggregate decision matrix $B^{r}$ of each decision-maker into a collective decision matrix $C B=\left[b_{h k}\right]_{m \times n}$.

Step 3: Calculate the ranking result by IMVN-PT-MULTIMOORA method.

Step 3.1: Conduct the IMVN-PT-ratio system method.

Based on the distance measure and Eq. (22)-(24), we can obtain the weights of criteria $Q$. Then, utilizing Eq. (20)-(21), we can derive the prospect value and the reference point. Finally, the ranking result can be obtained by Eq. (25)-(26).

Step 3.2: Conduct the IMVN-PT-reference point method.

According to Eq. (27)-(29), the ranking result can be obtained.

Step 3.3: Conduct the IMVN-PT-full multiplicative form method.

According to Eq. (30)-(31), the ranking result can be obtained.

Step 4: Calculate the final ranking result.

The dominance theory is employed to collect three ranking results of MULTIMOORA method.

\section{Case study}

The following case applies the IMVN-PT-MULTIMOORA method to deal with the MCGDM problem of selecting an appropriate scheme for subway construction. This case demonstrates the validity and advantages of the proposed method by sensitive analysis and comparative analysis.

Owing to improvements in quality of life, and a great deal of families possess their own cars, the problem of traffic jams is becoming prominent. The subway has some advantages, such as convenience, speediness and punctuality. When a subway system needs to be constructed, the government invites a group of experts to select one from four alternatives denoted as $A_{1}, A_{2}, A_{3}, A_{4}$. Considering the limited knowledge of each expert, we choose several experts to form an expert group $D=\left\{D_{1}, D_{2}, D_{3}, D_{4}, D_{5}\right\}$. In addition, the selected experts need not only good professional knowledge but also rich practical experience. The information of experts is shown in Table 1. Based on literature review $[59,60]$ and consulting some experts in this field, the following factors need to be considered: technology level $\left(C_{1}\right)$, environmental conditions $\left(C_{2}\right)$, the risk of 
public intervention $\left(C_{3}\right)$, and force of supervision $\left(C_{4}\right)$.

\subsection{Steps of the proposed method}

Step 1: Tidy up original data.

Firstly, in order to prevent the personal opinions of experts from being influenced by other experts in the process of scheme evaluation. All construction plans will be sent to experts by email. At the same time, in order to make the evaluation results as accurate as possible, some background information should be provided to the expert group. Then, evaluation information is obtained from experts. Next, the evaluation information about each scheme under criterion is transformed into MVNNs, as shown in Table 2-Table 6. Then, each evaluation matrix from expert is normalized according to Eq. (32). The normalized evaluation matrix is represented as $B^{r}=\left[b_{h k}\right]_{4 \times 4}(h, k=1,2,3,4)$.

Step 2: Obtain the collective decision matrix by IGMVNWHM operator.

According to Eq. (14), we can aggregate evaluation matrix $B^{r}$ of each decision-maker to obtain a collective evaluation matrix $C B=\left[b_{h k}\right]_{m \times n}$ where $s=t=1$. The weight vector of experts is subjectively determined, which is represented as $\phi=(0.1,0.2,0.2,0.25,0.25)$.

The final collective evaluation matrix is presented in Table 7.

Step 3: Calculate the ranking result by IMVN-PT-MULTIMOORA method.

Step 3.1: Conduct the IMVN-PT-ratio system method.

Firstly, the optimistic values of each criterion is determined.

$$
\begin{aligned}
& B^{+}=(\langle\{0.850,0.860\},\{0.150\},\{0.100\}\rangle,\langle\{0.860,0.878\},\{0.100,0.110\},\{0.100\}\rangle, \\
& \langle\{0.884\},\{0.319\},\{0.237,0.246\}\rangle,\langle\{0.844,0.870\},\{0.200\},\{0.100\}\rangle) \\
& B^{-}=(\langle\{0.456,0.475\},\{0.100\},\{0.100\}\rangle,\langle\{0.423,0.436\},\{0.100\},\{0.200\}\rangle, \\
& \langle\{0.630,0.651\},\{0.401\},\{0.287\}\rangle,\langle\{0.450,0.475\},\{0.100\},\{0.279\}\rangle)
\end{aligned}
$$

Then, according to the distance measure represented in Definition 7, we can get the distances between evaluation value of each criterion and optimistic/pessimistic values. In this paper, we assume $\mu=1$.

$$
\begin{aligned}
& d_{1}^{+}=0.382, d_{2}^{+}=0.384, d_{3}^{+}=0.148 \text { and } d_{4}^{+}=0.419 . \\
& d_{1}^{-}=0.457, d_{2}^{-}=0.453, d_{3}^{-}=0.234 \text { and } d_{4}^{-}=0.454 .
\end{aligned}
$$

Thus, the weights of criteria are obtained below:

$Q=(0.255,0.258,0.217,0.270)$. 
According to the investigation and experiment of Kalneman and Tversky [61]. We set $\alpha=\beta=0.88, \lambda=2.25, \gamma=0.61, \delta=0.69$. The ranking result is shown in Table 8 .

Step 3.2: Conduct the IMVN-PT-reference point method.

According to Eq. (27)-(29), the ranking result is shown in Table 8.

Step 3.3: Conduct the IMVN-PT-full multiplicative form method.

According to Eq. (30)-(31), the ranking result is presented below.

Step 4: Calculate the final ranking result.

Based on the dominance theory, the final ranking result is $A_{1} \succ A_{3} \succ A_{2} \succ A_{4}$.

\subsection{Influence of the parameter}

To obtain the effect of distinct parameter $\lambda$ on the result of this decision-making process, we conduct this analysis.

Firstly, we let $\lambda$ vary from 1 to 3 . Then, the results of IMVN-PT-ratio system, IMVN-PT-reference point and IMVN-PT-full multiplicative form method are shown in Table 9. Finally, we graph the results in Figure 2-Figure 4.

From Table 9 and Figure 2-Figure 4, we can find that the results obtained by three parts of IMVN-PT-MULTIMOORA method decrease with the increase of parameter $\lambda$. The ranking results has not changed with the change of parameter $\lambda$. The optimal alternative is always $A_{1}$. In Figure 2 , with the change of parameter $\lambda$, the numerical result of $A_{1}$ is larger than that of $A_{3}$ at first, and then smaller than that of $A_{3}$. In addition, the gap of numerical results between $A_{1}$ and $A_{3}$ decrease with the parameter $\lambda$ increase in Figure 3 and Figure 4. On this basis, we take a test on the parameter $\lambda$, when the value of parameter $\lambda$ is close to $34, A_{3}$ is optimal alternative. This means that when the decision-makers are more and more sensitive to the loss, the optimal alternative is more inclined to $A_{3}$. It also proves that it is necessary to introduce prospect theory into multi-criteria decision-making.

\subsection{Comparative analysis}

\subsubsection{Validity of the proposed method}

Given that the proposed method connects HM aggregation operator, prospect theory and MULTIMOORA method, we can select some existing methods based on these theories and methods.

In the method presented by $\mathrm{Li}$ et al. [62], some single-valued neutrosophic number HM operators, 
including the NNIGWHM and NNIGWGHM operators, are presented to integrate criterion values. Then, schemes will be ranked based on the values of the score and accuracy functions.

Tian et al. [48] presented an improved MULTIMOORA method for MCDM problem based on the closeness coefficient of TOPSIS and variation coefficient method. In addition, they defined distance measure for neutrosophic linguistic sets.

In order to conduct the method proposed by the above researchers, MVNNs should be transformed into SVNNs. We can derive SVNNs by calculating the average values of all possible truth-membership, indeterminacy-membership and falsity-membership degrees in MVNNs. For example, $a=\langle\{0.2,0.3\},\{0.3,0.5\},\{0.4,0.5\}\rangle$ can be transformed into $a_{1}=\langle 0.25,0.4,0.45\rangle$.

The ranking results obtained by $\mathrm{Li}$ et al. [62], Tian et al. [48] and the proposed method are shown in Table 10.

\subsubsection{Advantages of the proposed method}

Ji et al. [52] defined the operations of MVNSs and comparison methods. At the same time, some aggregation operators are presented to solve MCDM problems. To show the advantages of proposed method, we select WA and WG operators in this paper as an example. The results of this comparison will be shown in Table 11.

Pranab et al. [63] presented a novel TOPSIS-based approach for MCGDM problem in single-valued neutrosophic environments. In this paper, the authors obtain the evaluation information by linguistic terms. Then, an objective method is used to obtain the weight vector of each decision-maker.

Ji et al. [64] defined the projection measure of MVNNs, then, presented a projection-based TODIM method and applied it into personnel selection.

Based on the above, we conduct a comparison, the results of which are presented in Table 11.

Table 11 indicates that $A_{1}$ is always the best construction scheme for subways, while the worst scheme is $A_{4}$ or $A_{2}$. The proposed method produced the same ranking as the WA operator and TOPSIS method. At the same time, rankings derived from the proposed method have little difference with the WG operator and projection-based TODIM method. From Table 11, we can also conclude that our method is more reasonable than the WA, WG operators, TOPSIS and projection-based TODIM method. The reasons are as follows.

Although the WA operator produced the same ranking as the proposed method, the proposed method considers the interactions of the inputs. The WG operator also did not consider the interactions of the inputs. The TOPSIS and projection-based TODIM method ignore the bounded rationality of decision-makers. The 
proposed method utilizes IGMVNWHM operator to aggregate the assessments of all experts. Then, the collective decision matrix is input into the IMVN-PT-MULTIMOORA method to deal with practical problems better.

\section{Conclusion}

To address fuzzy and uncertain information in the process of selecting construction schemes for subways, MVNNs, HM operator, prospect theory and MULTIMOORA method in this decision-making problem are related. In this paper, we firstly introduced some basic concepts and theorems about MVNSs, HM operator. Then, the IGMVNWHM operator based on HM operator is presented. It considers the interactions among inputs. Subsequently, the new distance measure is defined between two MVNNs. Then, motived by TOPSIS and the variation coefficient method, we propose an improved MULTIMOORA method. Considering that decision-makers are not completely rational, we introduce the prospect theory into this method. Finally, a solution framework to select construction schemes for subways in multi-valued neutrosophic environments is developed. In addition, an application example is introduced to prove the validity and advantages of the proposed method. At the same time, we analyzed the rankings as the parameters changed.

The contributions and innovations of this paper are described as follows. First, MVNNs were used to present assessments of construction schemes for subways. Second, IGMVNWHM operator is introduced, which considers the interactions of inputs. Third, a new distance measure is defined between two MVNNs. Fourth, an IMVN-PT-MULTIMOORA method is presented.

In the future, the IGMVNWHM operator can be employed in other neutrosophic environments, such as probabilistic multi-valued neutrosophic sets. At the same time, we can explore other methods to solve multi-valued neutrosophic evaluation information problems.

\section{Acknowledgements}

The authors thank the editors and anonymous reviewers for their helpful comments and suggestions. This work was supported by the National Natural Science Foundation of China (Nos. 71901226 and 71871228), and Hunan Provincial Natural Science Foundation of China (Nos. 2019JJ51001).

\section{Reference}

1. Chen, Y.C., Hsu, L.H., and Tan, J.J.M. "A recursively construction scheme for super fault-tolerant hamiltonian graphs", Applied Mathematics \& Computation, 177(2), pp. 465-481 (2006).

2. Wang, Y., Wang, J.Q., and Wang, T.L. "Fuzzy stochastic multi-criteria decision-making methods with interval neutrosophic probability based on regret theory", Journal of Intelligent \& Fuzzy Systems, 35(2), pp. 2309-2322 (2018).

3. Ji, P., Wang, J.Q., and Zhang, H.Y. "Frank prioritized Bonferroni mean operator with single-valued neutrosophic sets and its application in selecting third-party logistics providers", Neural Computing \& 
Applications, 30(3), pp. 1-25 (2018).

4. Zadeh, L.A. "Fuzzy sets", Information \& Control, 8(3), pp. 338-353 (1965).

5. Luo, S.Z., Zhang, H.Y., Wang, J.Q., et al. "Group decision-making approach for evaluating the sustainability of constructed wetlands with probabilistic linguistic preference relations", Journal of the Operational Research Society, 70(12), pp. 2039-2055 (2018).

6. Nie, R.X., Tian, Z.P., Wang, J.Q., et al. "Pythagorean fuzzy multiple criteria decision analysis based on Shapley fuzzy measures and partitioned normalized weighted Bonferroni mean operator", International Journal of Intelligent Systems, 34(2), pp. 297-324 (2018).

7. Shao, X., Si, H., and Zhang, W. "Fuzzy wavelet neural control with improved prescribed performance for MEMS gyroscope subject to input quantization", Fuzzy Sets and Systems, 411, pp. 136-154 (2021).

8. Wang, X.K., Zhang, H.Y., Wang, J.Q., et al. "Extended TODIM-PROMETHEE II method with hesitant probabilistic information for solving potential risk evaluation problems of water resource carrying capacity", Expert Systems, pp. e12681 (2021).

9. Wang, X.K., Wang, S.H., Zhang, H.Y., et al. "The recommendation method for hotel selection under traveller preference characteristics: A cloud-based multi-criteria group decision support model", Group Decision and Negotiation, https://doi.org/10.1007/s10726-021-09735-0.

10. Atanassov, K.T. "Intuitionistic fuzzy sets", Fuzzy Sets \& Systems, 20(1), pp. 87-96 (1986).

11. Atanassov, K.T. "Two theorems for intuitionistic fuzzy sets", Fuzzy Sets \& Systems, 110(2), pp. 267-269 (2000).

12. Atanassov, K.T. "New operations defined over the intuitionistic fuzzy sets", Fuzzy Sets \& Systems, 61(2), pp. 137-142 (1994).

13. Ghosh, S.K., Biswas, B., and Ghosh, A. "Development of intuitionistic fuzzy special embedded convolutional neural network for mammography enhancement", Computational Intelligence, 37(1), pp. 47-69 (2021).

14. Kuo, R.J., Cheng, W.C., Lien, W.-C., et al. "Application of genetic algorithm-based intuitionistic fuzzy neural network to medical cost forecasting for acute hepatitis patients in emergency room", Journal of Intelligent \& Fuzzy Systems, 37(4), pp. 5455-5469 (2019).

15. Hu, J.H., Pan, L., Yang, Y., et al. "A group medical diagnosis model based on intuitionistic fuzzy soft sets", Applied Soft Computing, 77, pp. 453-466 (2019).

16. Atanassov, K. "Interval valued intuitionistic fuzzy sets", Fuzzy Sets \& Systems, 31(3), pp. 343-349 (1989).

17. Li, J. and Wang, J.Q. "Multi-criteria decision making with probabilistic hesitant fuzzy information based on expected multiplicative consistency", Neural Computing and Applications, 31(12), pp. 8897-8915 (2018).

18. Hu, J., Yang, Y., Zhang, X., et al. "Similarity and entropy measures for hesitant fuzzy sets", International Transactions in Operational Research, (2017).

19. Mishra, A.R., Rani, P., Krishankumar, R., et al. "An extended fuzzy decision-making framework using hesitant fuzzy sets for the drug selection to treat the mild symptoms of Coronavirus Disease 2019 (COVID-19)", Applied soft computing, 103, pp. 107155 (2021).

20. Zhu, B., Xu, Z., and Xia, M. "Dual Hesitant Fuzzy Sets", Journal of Applied Mathematics, 11(3), pp. 1-13 (2012).

21. Qian, G., Wang, H., and Feng, X. "Generalized hesitant fuzzy sets and their application in decision support system", Knowledge-Based Systems, 37(4), pp. 357-365 (2013).

22. Wang, H., Smarandache, F., Zhang, Y., et al. "Single valued neutrosophic sets", Review of the Air Force Academy, 10(2010).

23. Smarandache, F. "A unifying field in logics: Neutrosophic logic", Multiple-Valued Logic, 8(3), pp. 489-503 (1999). 
24. Smarandache, F. "Neutrosophic Set is a Generalization of Intuitionistic Fuzzy Set, Inconsistent Intuitionistic Fuzzy Set (Picture Fuzzy Set, Ternary Fuzzy Set), Pythagorean Fuzzy Set (Atanassov's Intuitionistic Fuzzy Set of second type), q-Rung Orthopair Fuzzy Set, Spherical Fuzzy Set, and n-HyperSpherical Fuzzy Set, while Neutrosophication is a Generalization of Regret Theory, Grey System Theory, and Three-Ways Decision (revisited)", Journal of New Theory, (29), pp. 1-31 (2019).

25. Rivieccio, U. "Neutrosophic logics: Prospects and problems", Fuzzy Sets \& Systems, 159(14), pp. 1860-1868 (2008).

26. Broumi, S., Bakali, A., Talea, M., et al. "Isolated Single Valued Neutrosophic Graphs", Neutrosophic Sets \& Systems, 11, pp. 74-78 (2016).

27. Sahin, R. and Kucuk, A. "Subsethood measure for single valued neutrosophic sets", Journal of Intelligent \& Fuzzy Systems, 29(2), pp. 525-530 (2015).

28. Li, Y.Y., Wang, J.Q., and Wang, T.L. "A linguistic neutrosophic multi-criteria group decision-making approach with EDAS method", Arabian Journal for Science and Engineering, 44(3), pp. 2737-2749 (2018).

29. Liang, R.X., Wang, J.Q., and Zhang, H.Y. "A multi-criteria decision-making method based on single-valued trapezoidal neutrosophic preference relations with complete weight information", Neural Computing and Applications, 30(11), pp. 3383-3398 (2017).

30. Tian, Z.P., Wang, J., Wang, J.Q., et al. "Simplified Neutrosophic Linguistic Multi-criteria Group Decision-Making Approach to Green Product Development", Group Decision \& Negotiation, 26(3), pp. 597-627 (2017).

31. Liu, P., Zhang, L., Liu, X., et al. "Multi-Valued Neutrosophic Number Bonferroni Mean Operators with their Applications in Multiple Attribute Group Decision Making", International Journal of Information Technology \& Decision Making, 15(5), pp. 1181-1210 (2016).

32. Peng, J.J., Wang, J.Q., and Yang, W.E. "A multi-valued neutrosophic qualitative flexible approach based on likelihood for multi-criteria decision-making problems", International Journal of Systems Science, 48(2), pp. 425-435 (2017).

33. Peng, J., Tian, C., Zhang, W., et al. "An integrated multi-criteria decision-making framework for sustainable supplier selection under picture fuzzy environment", Technological and Economic Development of Economy, 26(3), pp. 573-598 (2020).

34. Wang, J., Wang, J.Q., Tian, Z.P., et al. "A multihesitant fuzzy linguistic multicriteria decision-making approach for logistics outsourcing with incomplete weight information", International Transactions in Operational Research, 25(9)(2017).

35. Song, M., Jiang, W., Xie, C., et al. "A New Interval Numbers Power Average Operator in Multiple Attribute Decision Making", International Journal of Intelligent Systems, 32(6), pp. 631-644 (2016).

36. Li, Y. and Wang, Q.S. "Research on hotel supply chain risk assessment with dual generalized triangular fuzzy Bonferroni mean operators", Journal of Intelligent \& Fuzzy Systems, 37(2), pp. 1953-1965 (2019).

37. Li, Z. and Wei, G. "Pythagorean fuzzy heronian mean operators in multiple attribute decision making and their application to supplier selection", International Journal of Knowledge-Based and Intelligent Engineering Systems, 23(2), pp. 77-91 (2019).

38. Ayub, S., Abdullah, S., Ghani, F., et al. "Cubic fuzzy Heronian mean Dombi aggregation operators and their application on multi-attribute decision-making problem", Soft Computing, 25(6), pp. 4175-4189 (2021).

39. Liu, P.D., Liu, J.L., and Merigo, J.M. "Partitioned Heronian means based on linguistic intuitionistic fuzzy numbers for dealing with multi-attribute group decision making", Applied Soft Computing, 62(10), pp. 395-422 (2018).

40. Peng, J.J., Wang, J.Q., Hu, J.H., et al. "Multi-criteria decision-making approach based on single-valued neutrosophic hesitant fuzzy geometric weighted choquet integral heronian mean operator", Journal of 
Intelligent \& Fuzzy Systems, 35(3), pp. 1-14 (2018).

41. Liu, P. and Zhang, L. "Multiple criteria decision making method based on neutrosophic hesitant fuzzy Heronian mean aggregation operators", Journal of Intelligent \& Fuzzy Systems, 32(1), pp. 303-319 (2017).

42. Liu, P.D. "Multiple attribute group decision making method based on interval-valued intuitionistic fuzzy power Heronian aggregation operators", Computers \& Industrial Engineering, 108, pp. 199-212 (2017).

43. Sellak, H., Ouhbi, B., Frikh, B., et al. "Expertise-based consensus building for MCGDM with hesitant fuzzy linguistic information", Information Fusion, 50, pp. 54-70 (2019).

44. Brauers, W.K.M. and Zavadskas, E.K. "Project management by multimoora as an instrument for transition economies", Ukio Technologinis Ir Ekonominis Vystymas, 16(1), pp. 5-24 (2010).

45. Hafezalkotob, A., Hafezalkotob, A., Liao, H.C., et al. "An overview of MULTIMOORA for multi-criteria decision-making: Theory, developments, applications, and challenges", Information Fusion, 51, pp. 145-177 (2019).

46. Zhao, H., You, J.X., and Liu, H.C. "Failure mode and effect analysis using MULTIMOORA method with continuous weighted entropy under interval-valued intuitionistic fuzzy environment", Soft Computing, 21(18), pp. 5355-5367 (2016).

47. Liu, H.C., Zhao, H., You, X.Y., et al. "Robot Evaluation and Selection Using the Hesitant Fuzzy Linguistic MULTIMOORA Method", Journal of Testing and Evaluation, 47(2), pp. 1405-1426 (2019).

48. Tian, Z.P., Wang, J., Wang, J.Q., et al. "An improved MULTIMOORA approach for multi-criteria decision-making based on interdependent inputs of simplified neutrosophic linguistic information", Neural Computing \& Applications, 28, pp. S585-S597 (2017).

49. Nabeeh, N.A., Abdel-Monem, A., and Abdelmouty, A. "A Hybrid Approach of Neutrosophic with MULTIMOORA in Application of Personnel Selection", Neutrosophic Sets and Systems, 30, pp. 1-21 (2019).

50. Mi, X.M., Liao, H.C., Liao, Y., et al. "GREEN SUPPLER SELECTION BY AN INTEGRATED METHOD WITH STOCHASTIC ACCEPTABILITY ANALYSIS AND MULTIMOORA", Technological and Economic Development of Economy, 26(3), pp. 549-572 (2020).

51. Wu, S.M., You, X.Y., Liu, H.C., et al. "Improving quality function deployment analysis with the cloud MULTIMOORA method", International Transactions in Operational Research, 27(3), pp. 1600-1621 (2020).

52. Ji, P., Zhang, H.Y., and Wang, J.Q. "A fuzzy decision support model with sentiment analysis for items comparison in e-commerce: The case study of PConline.com", IEEE Transactions on Systems, Man, and Cybernetics: Systems, 49(10), pp. 1993-2004 (2018).

53. Liu, P., Cheng, S., and Zhang, Y. "An Extended Multi-criteria Group Decision-Making PROMETHEE Method Based on Probability Multi-valued Neutrosophic Sets", International Journal of Fuzzy Systems, 21(2), pp. 388-406 (2019).

54. Liu, P.D., Cheng, S.F., and Zhang, Y.M. "An Extended Multi-criteria Group Decision-Making PROMETHEE Method Based on Probability Multi-valued Neutrosophic Sets", International Journal of Fuzzy Systems, 21(2), pp. 388-406 (2019).

55. Liu, P. and Li, Y. "An extended MULTIMOORA method for probabilistic linguistic multi-criteria group decision-making based on prospect theory", Computers \& Industrial Engineering, 136(2019).

56. Liu, P. and Chen, S.M. "Group Decision Making Based on Heronian Aggregation Operators of Intuitionistic Fuzzy Numbers", IEEE Trans Cybern, 47(9), pp. 2514-2530 (2017).

57. Sumi and Hiroki "The space of \$2\$-generator postcritically bounded polynomial semigroups and random complex dynamics", Advances in Mathematics, 290, pp. 809-859 (2014).

58. Wang, T.X., Li, H.X., Zhang, L.B., et al. "A three-way decision model based on cumulative prospect theory", Information Sciences, 519, pp. 74-92 (2020). 
59. Ghanbaripour, A.N., Sher, W., and Yousefi, A. "Critical success factors for subway construction projects main contractors' perspectives", International Journal of Construction Management, 20(3), pp. 177-195 (2020).

60. Nouri, F., Khorasani-Zavareh, D., and Mohammadi, R. "Factor's affecting safe emergency evacuation of subways in Iran: findings of a qualitative study", Journal of injury \& violence research, 12(2)(2020).

61. Tversky, K.A. "Prospect Theory: an Analysis of Decision under Risk", Econometrica, 47(2), pp. 263-291 (1979).

62. Yanhua, L.I., Liu, P., and Chen, Y. "Some Single Valued Neutrosophic Number Heronian Mean Operators and Their Application in Multiple Attribute Group Decision Making", Informatica, 27(1), pp. 85-110 (2014).

63. Pramanik, S., Pramanik, S., and Giri, B.C. "TOPSIS method for multi-attribute group decision-making under single-valued neutrosophic environment", Neural Computing \& Applications, 27(3), pp. 727-737 (2016).

64. Ji, P., Zhang, H.Y., and Wang, J.Q. "A projection-based TODIM method under multi-valued neutrosophic environments and its application in personnel selection", Neural Computing \& Applications, 29(1), pp. 1-14 (2017).

Fei Xiao is a $\mathrm{PhD}$ degree candidate in management science and engineering at the Business School of Central South University. His research interests include information management, machine learning and decision-making theory and applications. E-mail: xiaofei2017@csu.edu.cn

Jing Wang is an associate professor in the College of Tourism, Hunan Normal University, China. She received her PhD degree in Management Science and Engineering, from the School of Business, Central South University in 2016. She also holds an MSc degree in Information Engineering from University of Osnabrueck, Germany, in 2006. Her current research focuses on decision-making theory and applications, and quality management. E-mail: wangjing@csuft.edu.cn

Jian-qiang Wang is a professor in the Department of Management Science and Information Management at the Business School of Central South University. He holds a PhD in management science \& engineering and he is also a $\mathrm{PhD}$ supervisor in this major. Over the past couple decades, his research interests are in the area of decision-making theory. His current research interests include information management, and decision-making theory and applications, and risk management and control. E-mail: jqwang@csu.edu.cn

Figure 1. Solution framework for MVN-MCGDM problem.

Figure 2. Results obtained by IMVN-PT-ratio system method.

Figure 3. Results obtained by IMVN-PT-reference point method.

Figure 4. Results obtained by IMVN-PT-full multiplicative form method.

Table 1. Information of experts.

Table 2. Evaluation information collected from expert 1.

Table 3. Evaluation information collected from expert 2. 
Table 4. Evaluation information collected from expert 3.

Table 5. Evaluation information collected from expert 4.

Table 6. Evaluation information collected from expert 5.

Table 7. Collective evaluation matrix.

Table 8. Ranking results obtained by proposed method.

Table 9. Results obtained by different values of $\lambda$.

Table 10. Ranking results derived from different methods.

Table 11. Ranking results obtained by different methods.

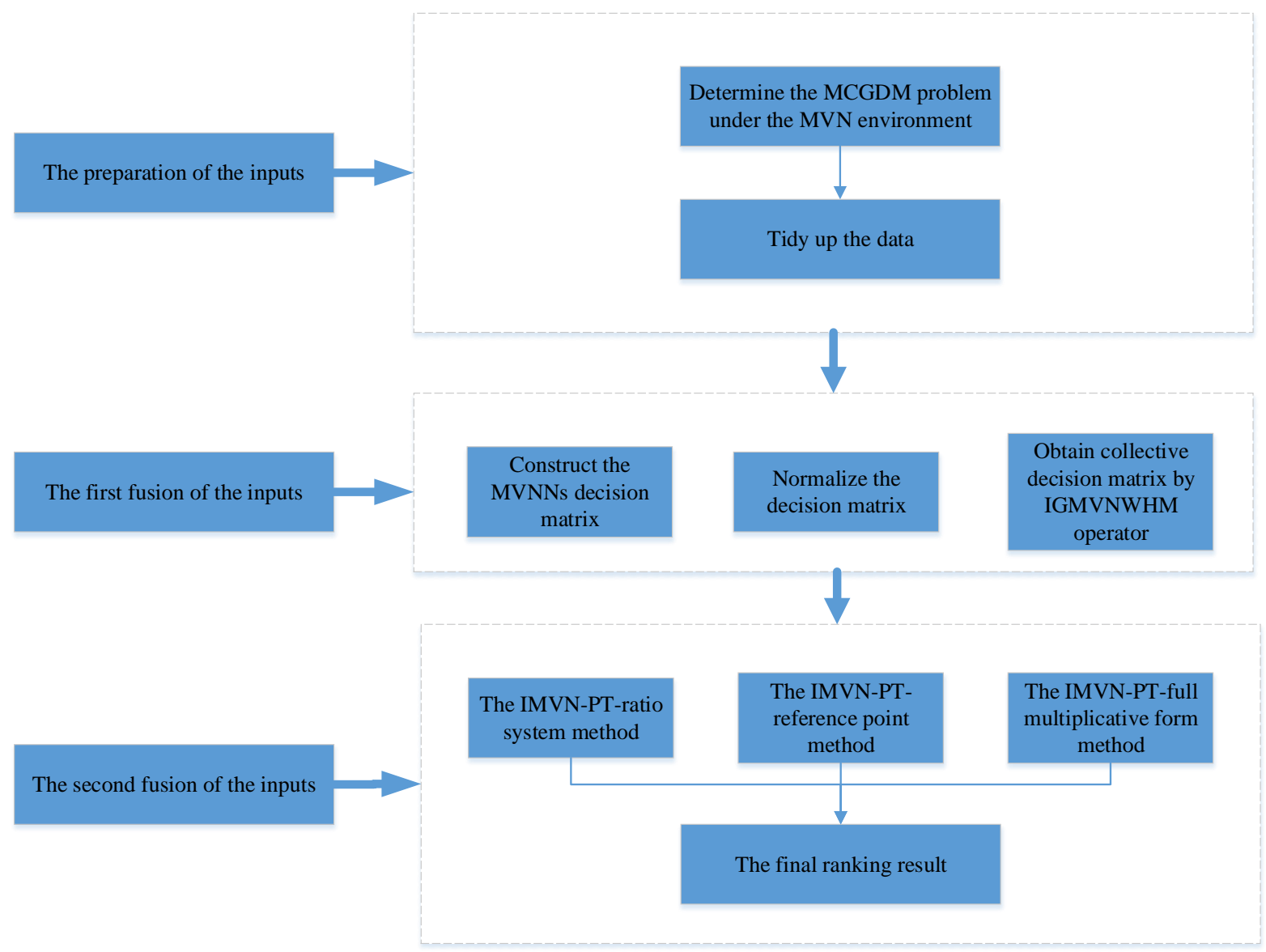

Figure 1. Solution framework for MVN-MCGDM problem. 


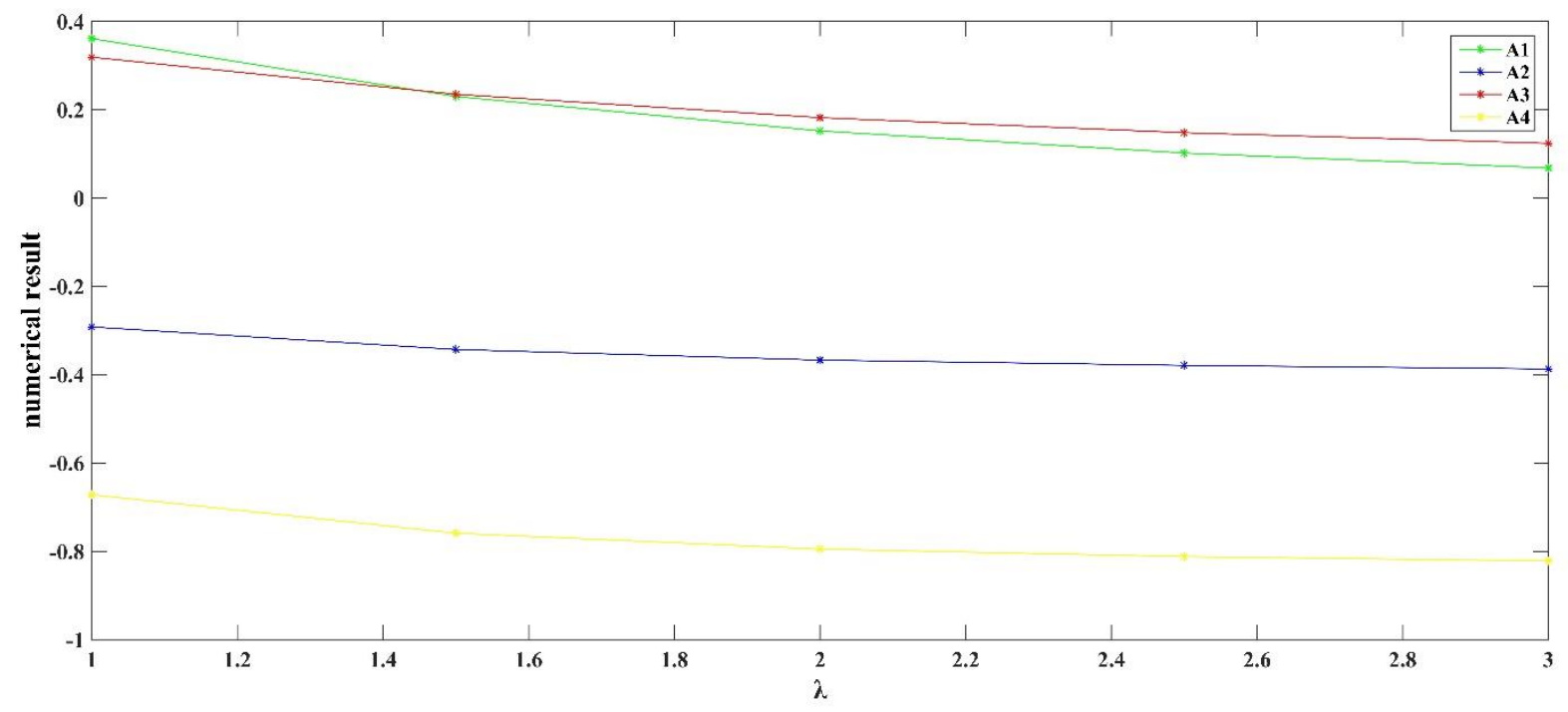

Figure 2. Results obtained by IMVN-PT-ratio system method.

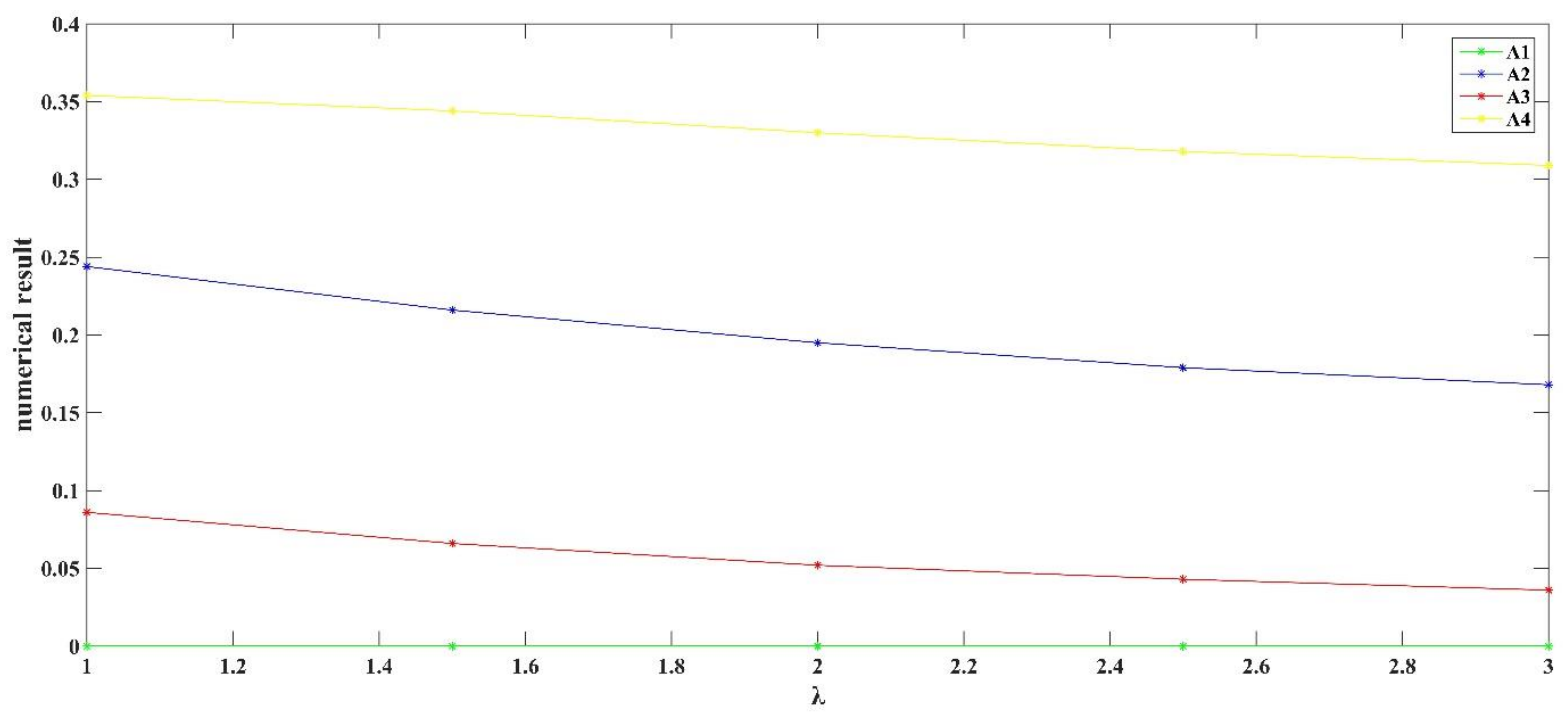

Figure 3. Results obtained by IMVN-PT-reference point method.

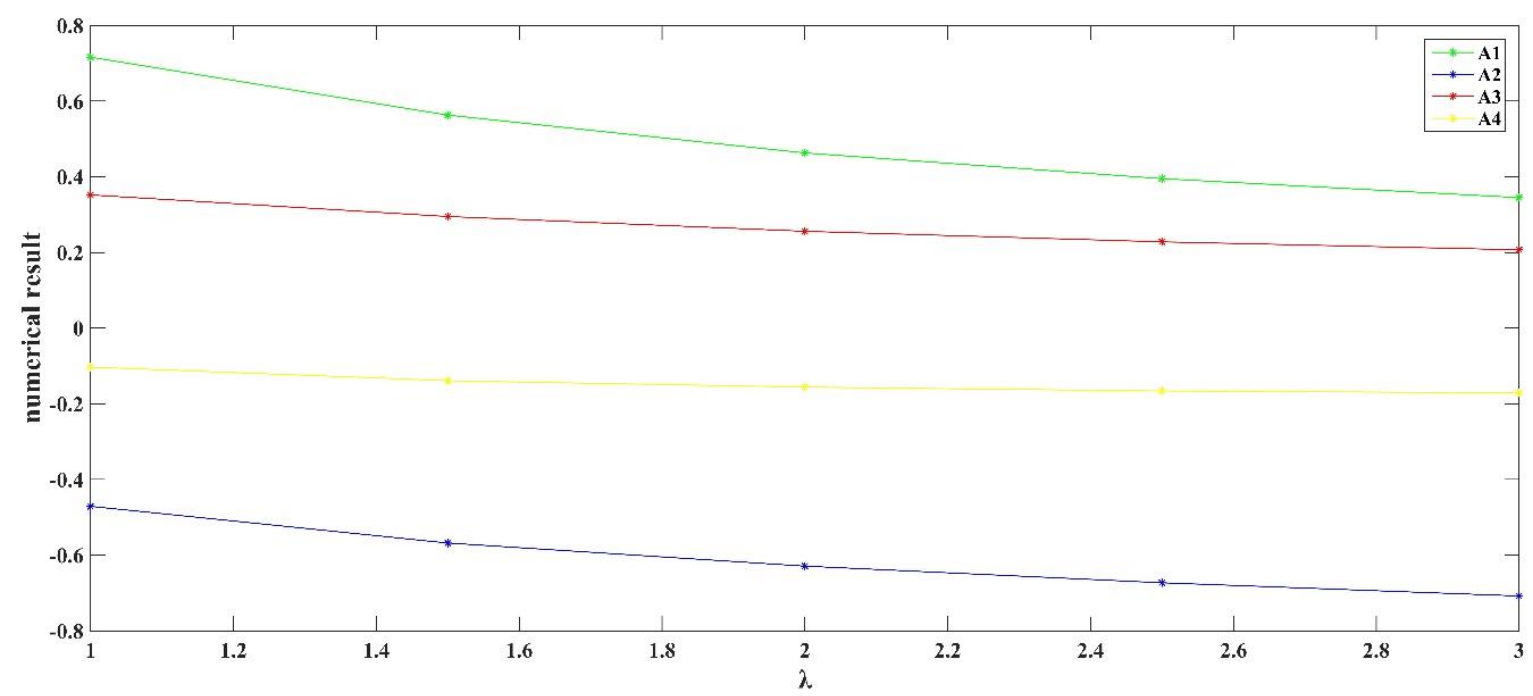


Figure 4. Results obtained by IMVN-PT-full multiplicative form method.

Table 1. Information of experts.

\begin{tabular}{ccccc}
\hline Experts & Education & Positional titles & $\begin{array}{c}\text { Employment } \\
\text { position }\end{array}$ & Working years \\
\hline Expert 1 & M.S. & Engineer & Project manager & 12 \\
Expert 2 & M.S. & Engineer & Technical manager & 15 \\
Expert 3 & Ph.D. & Senior engineer & Economic manager & 15 \\
Expert 4 & Ph.D. & Senior engineer & Risk manager & 18 \\
Expert 5 & Ph.D. & Senior engineer & General manager & 20 \\
\hline
\end{tabular}

Table 2. Evaluation information collected from expert 1.

\begin{tabular}{ccccc}
\hline & $C_{1}$ & $C_{2}$ & $C_{3}$ & $C_{4}$ \\
\hline$A_{1}$ & $\langle\{0.8,0.9\},\{0.2\},\{0.1\}\rangle$ & $\langle\{0.9\},\{0.1\},\{0.1\}\rangle$ & $\langle\{0.4\},\{0.8\},\{0.7\}\rangle$ & $\langle\{0.8\},\{0.2\},\{0.1\}\rangle$ \\
$A_{2}$ & $\langle\{0.6,0.7\},\{0.3\},\{0.3\}\rangle$ & $\langle\{0.6\},\{0.3\},\{0.1\}\rangle$ & $\langle\{0.2\},\{0.6\},\{0.7\}\rangle$ & $\langle\{0.6\},\{0.1\},\{0.2\}\rangle$ \\
$A_{3}$ & $\langle\{0.7\},\{0.2\},\{0.1\}\rangle$ & $\langle\{0.8\},\{0.1\},\{0.2\}\rangle$ & $\langle\{0.2,0.3\},\{0.7\},\{0.9\}\rangle$ & $\langle\{0.7\},\{0.2\},\{0.2\}\rangle$ \\
$A_{4}$ & $\langle\{0.5\},\{0.1\},\{0.1\}\rangle$ & $\langle\{0.4,0.5\},\{0.1\},\{0.2\}\rangle$ & $\langle\{0.2\},\{0.7\},\{0.6\}\rangle$ & $\langle\{0.5\},\{0.1\},\{0.3\}\rangle$ \\
\hline
\end{tabular}

Table 3. Evaluation information collected from expert 2.

\begin{tabular}{ccccc}
\hline & $C_{1}$ & $C_{2}$ & $C_{3}$ & $C_{4}$ \\
\hline$A_{1}$ & $\langle\{0.8\},\{0.1\},\{0.1\}\rangle$ & $\langle\{0.8,0.9\},\{0.1\},\{0.1\}\rangle$ & $\langle\{0.4\},\{0.8\},\{0.7\}\rangle$ & $\langle\{0.9\},\{0.2\},\{0.1\}\rangle$ \\
$A_{2}$ & $\langle\{0.7\},\{0.3\},\{0.3\}\rangle$ & $\langle\{0.6\},\{0.2\},\{0.1\}\rangle$ & $\langle\{0.2\},\{0.6\},\{0.6\}\rangle$ & $\langle\{0.6\},\{0.1,0.2\},\{0.2\}\rangle$ \\
$A_{3}$ & $\langle\{0.8\},\{0.2\},\{0.1\}\rangle$ & $\langle\{0.8\},\{0.1\},\{0.1\}\rangle$ & $\langle\{0.3\},\{0.6\},\{0.8\}\rangle$ & $\langle\{0.7\},\{0.2\},\{0.1\}\rangle$ \\
$A_{4}$ & $\langle\{0.5\},\{0.1\},\{0.1\}\rangle$ & $\langle\{0.4\},\{0.1\},\{0.2\}\rangle$ & $\langle\{0.3\},\{0.7\},\{0.6\}\rangle$ & $\langle\{0.5\},\{0.1\},\{0.3\}\rangle$ \\
\hline
\end{tabular}

Table 4. Evaluation information collected from expert 3.

$\begin{array}{cccc}C_{1} & C_{2} & C_{3} & C_{4}\end{array}$




\begin{tabular}{lcccc}
$A_{1}$ & $\langle\{0.9\},\{0.2\},\{0.1\}\rangle$ & $\langle\{0.9\},\{0.1,0.2\},\{0.1\}\rangle$ & $\langle\{0.2\},\{0.8\},\{0.6,0.7\}\rangle$ & $\langle\{0.9\},\{0.2\},\{0.1\}\rangle$ \\
$A_{2}$ & $\langle\{0.7\},\{0.3\},\{0.3\}\rangle$ & $\langle\{0.6,0.7\},\{0.3\},\{0.1\}\rangle$ & $\langle\{0.2\},\{0.6\},\{0.7\}\rangle$ & $\langle\{0.6\},\{0.1\},\{0.2\}\rangle$ \\
$A_{3}$ & $\langle\{0.6,0.7\},\{0.2\},\{0.1\}\rangle$ & $\langle\{0.7\},\{0.1\},\{0.2\}\rangle$ & $\langle\{0.3\},\{0.7\},\{0.9\}\rangle$ & $\langle\{0.7\},\{0.2\},\{0.1\}\rangle$ \\
$A_{4}$ & $\langle\{0.4,0.5\},\{0.1\},\{0.1\}\rangle$ & $\langle\{0.4\},\{0.1\},\{0.2\}\rangle$ & $\langle\{0.2\},\{0.7\},\{0.6\}\rangle$ & $\langle\{0.5\},\{0.1\},\{0.2\}\rangle$ \\
\hline
\end{tabular}

Table 5. Evaluation information collected from expert 4.

\begin{tabular}{ccccc}
\hline & $C_{1}$ & $C_{2}$ & $C_{3}$ & $C_{4}$ \\
\hline$A_{1}$ & $\langle\{0.9\},\{0.1\},\{0.1\}\rangle$ & $\langle\{0.8\},\{0.1\},\{0.1\}\rangle$ & $\langle\{0.2\},\{0.5\},\{0.6\}\rangle$ & $\langle\{0.8\},\{0.2\},\{0.1\}\rangle$ \\
$A_{2}$ & $\langle\{0.6\},\{0.3\},\{0.3\}\rangle$ & $\langle\{0.6\},\{0.3\},\{0.1\}\rangle$ & $\langle\{0.2,0.3\},\{0.6\},\{0.7\}\rangle$ & $\langle\{0.6\},\{0.2\},\{0.3\}\rangle$ \\
$A_{3}$ & $\langle\{0.7\},\{0.2\},\{0.1\}\rangle$ & $\langle\{0.8\},\{0.1\},\{0.2\}\rangle$ & $\langle\{0.2\},\{0.7\},\{0.9\}\rangle$ & $\langle\{0.7,0.8\},\{0.2\},\{0.2\}\rangle$ \\
$A_{4}$ & $\langle\{0.5\},\{0.1\},\{0.1\}\rangle$ & $\langle\{0.5\},\{0.1\},\{0.2\}\rangle$ & $\langle\{0.2\},\{0.7\},\{0.6\}\rangle$ & $\langle\{0.4\},\{0.1\},\{0.3\}\rangle$ \\
\hline
\end{tabular}

Table 6. Evaluation information collected from expert 5.

\begin{tabular}{ccccc}
\hline & $C_{1}$ & $C_{2}$ & $C_{3}$ & $C_{4}$ \\
\hline$A_{1}$ & $\langle\{0.8\},\{0.2\},\{0.1\}\rangle$ & $\langle\{0.9\},\{0.1\},\{0.1\}\rangle$ & $\langle\{0.2\},\{0.4\},\{0.6\}\rangle$ & $\langle\{0.8,0.9\},\{0.2\},\{0.1\}\rangle$ \\
$A_{2}$ & $\langle\{0.6\},\{0.3\},\{0.3\}\rangle$ & $\langle\{0.6\},\{0.3\},\{0.1\}\rangle$ & $\langle\{0.2\},\{0.6\},\{0.7\}\rangle$ & $\langle\{0.6\},\{0.1\},\{0.2\}\rangle$ \\
$A_{3}$ & $\langle\{0.8\},\{0.2\},\{0.1\}\rangle$ & $\langle\{0.8\},\{0.2\},\{0.2\}\rangle$ & $\langle\{0.2\},\{0.7\},\{0.9\}\rangle$ & $\langle\{0.8\},\{0.2\},\{0.2\}\rangle$ \\
$A_{4}$ & $\langle\{0.4\},\{0.1\},\{0.1\}\rangle$ & $\langle\{0.4\},\{0.1\},\{0.2\}\rangle$ & $\langle\{0.2\},\{0.7\},\{0.6\}\rangle$ & $\langle\{0.4,0.5\},\{0.1\},\{0.3\}\rangle$ \\
\hline
\end{tabular}

Table 7. Collective evaluation matrix.

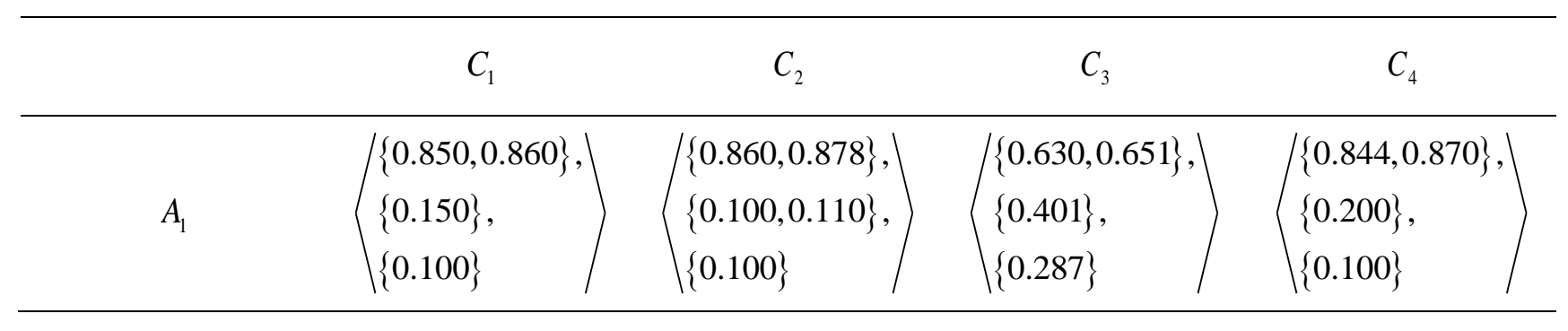




\begin{tabular}{|c|c|c|c|c|}
\hline$A_{2}$ & $\left\langle\begin{array}{l}\{0.641,0.651\}, \\
\{0.300\}, \\
\{0.300\}\end{array}\right\rangle$ & $\left(\begin{array}{l}\{0.600,0.621\}, \\
\{0.279\}, \\
\{0.123\}\end{array}\right)$ & $\left\langle\begin{array}{l}\{0.682\}, \\
\{0.400\}, \\
\{0.2,0.224\}\end{array}\right\rangle$ & $\left.\begin{array}{l}\{0.600\}, \\
\{0.122,0.141\}, \\
\{0.224\}\end{array}\right)$ \\
\hline$A_{3}$ & $\left\langle\begin{array}{l}\{0.732,0.748\}, \\
\{0.200\}, \\
\{0.100\}\end{array}\right.$ & $\left\langle\begin{array}{l}\{0.782\}, \\
\{0.123\}, \\
\{0.177\}\end{array}\right\rangle$ & $\mid \begin{array}{l}\{0.884\}, \\
\{0.319\}, \\
\{0.237,0.246\}\end{array}$ & $\begin{array}{l}\{0.728,0.754\}, \\
\{0.200\}, \\
\{0.156\}\end{array}$ \\
\hline$A_{4}$ & $\left\langle\begin{array}{l}\{0.456,0.475\}, \\
\{0.100\}, \\
\{0.100\}\end{array}\right\rangle$ & $\left(\begin{array}{l}\{0.423,0.436\}, \\
\{0.100\}, \\
\{0.200\}\end{array}\right)$ & $\left(\begin{array}{l}\{0.600\}, \\
\{0.300\}, \\
\{0.218\}\end{array}\right)$ & $\begin{array}{l}\{0.450,0.475\}, \\
\{0.100\}, \\
\{0.279\}\end{array}$ \\
\hline
\end{tabular}

Table 8 . Ranking results obtained by proposed method.

\begin{tabular}{|c|c|c|c|c|c|c|}
\hline & \multicolumn{2}{|c|}{$\begin{array}{l}\text { IMVN-PT-ratio system } \\
\text { method }\end{array}$} & \multicolumn{2}{|c|}{$\begin{array}{l}\text { IMVN-PT-reference point } \\
\text { method }\end{array}$} & \multicolumn{2}{|c|}{$\begin{array}{l}\text { IMVN-PT-full multiplicative } \\
\text { form method }\end{array}$} \\
\hline & $\begin{array}{c}\text { Calculating } \\
\text { result }\end{array}$ & Rank & $\begin{array}{l}\text { Calculating } \\
\text { result }\end{array}$ & Rank & $\begin{array}{l}\text { Calculating } \\
\text { result }\end{array}$ & Rank \\
\hline$A_{1}$ & 0.125 & 1 & 0 & 1 & 0.426 & 1 \\
\hline$A_{2}$ & -0.374 & 3 & 0.186 & 3 & -0.653 & 4 \\
\hline$A_{3}$ & 0.164 & 2 & 0.047 & 2 & 0.241 & 2 \\
\hline$A_{4}$ & -0.805 & 4 & 0.324 & 4 & -0.162 & 3 \\
\hline
\end{tabular}

Table 9. Results obtained by different values of $\lambda$.

\begin{tabular}{cccccc}
\hline & & $A_{1}$ & $A_{2}$ & $A_{3}$ & $A_{4}$ \\
\hline & $y_{i}^{*}$ & 0.361 & -0.292 & 0.319 & -0.672 \\
& $z_{i}^{*}$ & 0 & 0.244 & 0.086 & 0.354 \\
& $u_{i}^{*}$ & 0.716 & -0.471 & 0.352 & -0.103 \\
& Ranking & 1 & 3 & 2 & 4 \\
& $y_{i}^{*}$ & 0.230 & -0.343 & 0.235 & -0.759 \\
& $z_{i}^{*}$ & 0 & 0.216 & 0.066 & 0.344 \\
\hline
\end{tabular}




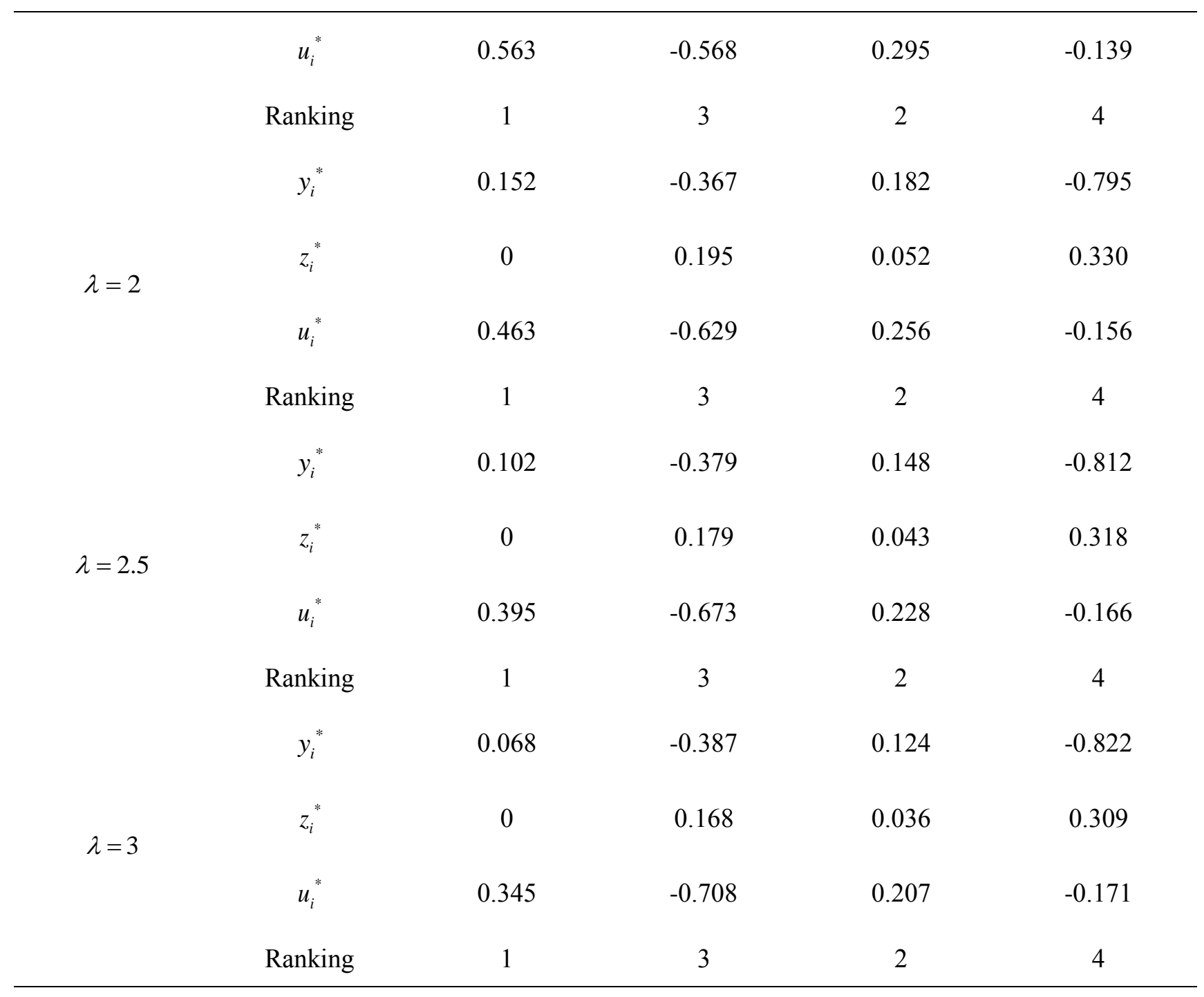

Table 10. Ranking results derived from different methods.

\begin{tabular}{cc}
\hline Method & Ranking \\
\hline NNIGWHM operator & $A_{1} \succ A_{3} \succ A_{4} \succ A_{2}$ \\
(presented by Li et al. [59]) & \\
NNIGWGHM operator & $A_{1} \succ A_{3} \succ A_{4} \succ A_{2}$ \\
(presented by Li et al. [59]) & \\
MULTIMOORA method & $A_{1} \succ A_{3} \succ A_{4} \succ A_{2}$ \\
(presented by Tian et al. [46]) & \\
The proposed method & $A_{1} \succ A_{3} \succ A_{4} \succ A_{2}$ \\
\hline
\end{tabular}

Table 11. Ranking results obtained by different methods. 
WA operator (presented by Ji et al. [52])

WG operator (presented by Ji et al. [52])

TOPSIS method (presented by Pranab et al. [63])

Projection-based TODIM method

(presented by Ji et al. [64])

The proposed method
$A_{1} \succ A_{3} \succ A_{2} \succ A_{4}$

$A_{1} \succ A_{3} \succ A_{4} \succ A_{2}$

$A_{1} \succ A_{3} \succ A_{2} \succ A_{4}$

$A_{1} \succ A_{3} \succ A_{4} \succ A_{2}$

$A_{1} \succ A_{3} \succ A_{2} \succ A_{4}$ 